\title{
A family of $C^{0}$ finite elements for Kirchhoff plates II: Numerical results
}

Lourenço Beirão da Veiga, Jarkko Niiranen, Rolf Stenberg

Helsinki University of Technology, Institute of Mathematics Research Reports, A526 (2007), 29 pages

accepted for publication in

Computer Methods in Applied Mechanics and Engineering.

Copyright (c) 2007 authors \& Elsevier B.V.

Preprinted with permission from Elsevier. 
Helsinki University of Technology, Institute of Mathematics, Research Reports Teknillisen korkeakoulun matematiikan laitoksen tutkimusraporttisarja

\section{A FAMILY OF $C^{0}$ FINITE ELEMENTS FOR KIRCHHOFF PLATES II: NUMERICAL RESULTS}

Lourenço Beirão da Veiga Jarkko Niiranen Rolf Stenberg 

Helsinki University of Technology, Institute of Mathematics, Research Reports Teknillisen korkeakoulun matematiikan laitoksen tutkimusraporttisarja

\section{A FAMILY OF $C^{0}$ FINITE ELEMENTS FOR KIRCHHOFF PLATES II: NUMERICAL RESULTS}

Lourenço Beirão da Veiga Jarkko Niiranen Rolf Stenberg 
Lourenço Beirão da Veiga, Jarkko Niiranen, Rolf Stenberg: A family of $C^{0}$ finite elements for Kirchhoff plates II: Numerical results; Helsinki University of Technology, Institute of Mathematics, Research Reports A526 (2007).

\begin{abstract}
A new family of $C^{0}$ Kirchhoff plate elements has been introduced by the authors in the theoretical counterpart of the present paper; A family of $C^{0}$ finite elements for Kirchhoff plates I: Error analysis [6]. The method presented is a displacement formulation with the deflection and the rotation as unknowns. In the theoretical part, an a-priori and an a-posteriori error analysis has been accomplished for the family. In the present contribution, the authors first recall the main theoretical results, and then focus on the computational aspects of the method, and finally present a set of numerical results on various benchmark computations. These tests verify the optimal convergence rate of the method and illustrate the robustness of the reliable and efficient residual based a-posteriori error estimator for adaptive mesh refinements.
\end{abstract}

AMS subject classifications: 65N30, 74S05, 74K20

Keywords: finite elements, Kirchhoff plate model, free boundary, a-priori error tests, a-posteriori error tests, adaptivity

\title{
Correspondence
}

beirao@mat.unimi.it,Jarkko.Niiranen@tkk.fi, Rolf.Stenberg@tkk.fi

ISBN 978-951-22-8815-1

Helsinki University of Technology

Department of Engineering Physics and Mathematics

Institute of Mathematics

P.O. Box 1100, FI-02015 TKK, Finland

email:math@tkk.fi http://math.tkk.fi/ 


\section{Introduction}

For linearly elastic plate structures, the most commonly used plate models in practical engineering applications are the Kirchhoff-Love and ReissnerMindlin models. The Reissner-Mindlin model is more accurate and usually preferred for moderately thin plates, whereas the simpler Kirchhoff-Love (or Kirchhoff) model becomes a valid option for thinner plate structures. In general, the choice of the model should rest on the smoothness of the solution for the loading and the boundary conditions considered, and on the goals of computations $[3,2,1]$. Especially, if the shear stress is smooth enough and the boundary effects are not of special interest, the Kirchhoff theory provides a model with a reasonable accuracy for the bending problem of a thin plate.

From the strict numerical point of view, the Kirchhoff model carries two non-negligible advantages. One is the presence of a single scalar field of unknowns, the deflections, without the additional vector field, the rotations, needed in the Reissner-Mindlin model. The second advantage is the higher regularity of the Kirchhoff solution, which allows, in principle, a more accurate finite element approximation.

On the other hand, the fundamental difficulty in designing a finite element method for the Kirchhoff plate bending problem originates from the corresponding variational formulation, since the natural variational space for the biharmonic problem is the second-order Sobolev space $H^{2}$. Thus, differently from the Reissner-Mindlin model, a conforming finite element approximation of the Kirchhoff problem requires globally $C^{1}$-continuous elements, such as the well known Argyris triangle [14], which imply a high polynomial order.

A viable choice in order to avoid using high-order polynomial spaces is to adopt non-standard finite elements, such as the Morley triangle [23, 14], Hsieh-Clough-Tocher triangle or the Discrete Kirchhoff Triangle [4, 14]. Another type of classical approach is the mixed finite element method presented in $[15]$.

A natural alternative is, instead, to write the problem as a limit of the Reissner-Mindlin problem written in mixed form. In the presence of free boundary conditions, however, this leads to a method which is not consistent: the solution of the Kirchhoff problem does not coincide with the solution of the Reissner-Mindlin problem with the thickness set equal to zero $[5,6]$. For the discretized problem, instead of the converge rate of order $\mathcal{O}\left(h^{k}\right)$, the inconsistency on the free boundary leads to an unexpected converge rate of order $\mathcal{O}\left(h^{1 / 2}\right)$, independently of the polynomial order $k[24,5]$.

A remedy to the boundary inconsistency is obtained in [16] by adding a term penalizing the Kirchhoff condition along the free boundaries. However, this approach requires a strong penalization leading to an ill-conditioned discrete system. Another type of modern approach of using $C^{0}$-continuous approximations is based on continuous-discontinuous Galerkin methods and stabilization techniques [17]. This approach, however, leads to bilinear forms with additional terms for all inter-element boundaries.

In the more recent approach [6], we present a family of $C^{0}$-continuous fi- 
nite elements for the Kirchhoff problem, which do not suffer from the boundary inconsistency mentioned above. The inconsistency on the free boundaries is treated by additional Nitsche-type terms in the bilinear form guaranteeing the consistency, symmetry and the stability of the method (cf. [26] as well). We emphasize that the additional terms concern the edges of the elements on free boundaries alone. This finite element method is a modification of the stabilized method for the Reissner-Mindlin plates in [25]. The family includes "simple low-order" elements and does not suffer from the conditioning problems of the one in [16]. The optimal convergence rate of the method, with respect to the solution regularity and the polynomial degree, is proved in [6] and it holds even in the presence of free boundaries. Furthermore, in [6] a local a-posteriori error indicator is presented and it is shown to be both reliable and efficient - reliable in the sense that it provides an upper bound for the true error, efficient in the sense that it gives a lower bound.

Our aim in the present paper is to accomplish a comprehensive numerical testing for the theoretical results derived in [6]. Therefore, we perform a wide range of benchmark computations, in order to verify the theoretical a-priori error estimates and illustrate the robustness of the residual based a-posteriori error estimator. The numerical tests comprise different kinds of convex and non-convex domains, boundary conditions and loadings. For this paper to be rather self-contained, we review the main results given in [6]. In particular, we give here a more detailed and constructive motivation for the method, starting from the first ideas by Hughes and Franca [19] of using Galerkinleast-squares (or stabilized) ideas for plates. Furthermore, we include the straightforward stability proof in the present paper.

It is further to be noted that in the literature there seems to occur no other a-posteriori error analysis for Kirchhoff plates than the one in [13] for the mixed Ciarlet-Raviart formulation with clamped boundaries, the analysis in [7] for the classical non-conforming Morley element [23, 14, 22] in the clamped case and our analysis in [6] for the present formulation with clamped, simply supported or free boundaries.

The paper is organized as follows. In the next Section, we recall the Kirchhoff plate bending model. In Section 3, we derive and present the new finite element family. Then, in Sections 4 and 5, respectively, we state the main results of the theoretical a-priori and a-posteriori error analysis of [6]. In Section 6, we finally present the numerical results, divided into an a-priori and an a-posteriori part, confirming the theory of the previous sections and the applicability of the method.

\section{The Kirchhoff plate bending problem}

We consider the bending problem of an isotropic linearly elastic plate under the transverse loading $g$. The midsurface of the undeformed plate is described by a convex polygonal domain $\Omega \subset \mathbb{R}^{2}$. The plate is considered to be clamped on the part $\Gamma_{\mathrm{C}}$ of its boundary $\partial \Omega$, simply supported on the part $\Gamma_{\mathrm{S}} \subset \partial \Omega$ 
and free on $\Gamma_{\mathrm{F}} \subset \partial \Omega$. With $\mathcal{V}$ we indicate the collection of all the corner points in $\Gamma_{\mathrm{F}}$ corresponding to an angle of the free boundary.

\subsection{The biharmonic formulation}

First, we define the material constants for the model: The bending modulus and the shear modulus, respectively, are denoted by

$$
\mathrm{D}=\frac{\mathrm{E} t^{3}}{12\left(1-\nu^{2}\right)} \quad \text { and } \quad \mathrm{G}=\frac{\mathrm{E}}{2(1+\nu)}
$$

with the Young modulus $\mathrm{E}$ and the Poisson ratio $\nu$ for the material. The thickness of the plate is denoted by $t$.

In the sequel, we need the following partial differential operators: The strain tensor $\varepsilon$ is defined as the symmetric tensor gradient

$$
\boldsymbol{\varepsilon}(\boldsymbol{\eta})=\left(\boldsymbol{\nabla} \boldsymbol{\eta}+(\boldsymbol{\nabla} \boldsymbol{\eta})^{T}\right) \quad \text { with } \quad \boldsymbol{\nabla} \boldsymbol{\eta}=\left(\begin{array}{ll}
\frac{\partial \eta_{x}}{\partial x} & \frac{\partial \eta_{x}}{\partial y} \\
\frac{\partial \eta_{y}}{\partial x} & \frac{\partial \eta_{y}}{\partial y}
\end{array}\right) .
$$

The vector gradient and the vector divergence are defined as usual, while the tensor divergence is defined as

$$
\operatorname{div} \boldsymbol{\sigma}=\left(\begin{array}{c}
\frac{\partial \sigma_{x x}}{\partial x}+\frac{\partial \sigma_{x y}}{\partial y} \\
\frac{\partial \sigma_{y x}}{\partial x}+\frac{\partial \sigma_{y y}}{\partial y}
\end{array}\right)
$$

Next, we define the physical quantities for the problem: The bending moment is defined as

$$
\boldsymbol{M}(\nabla w)=\mathrm{D}((1-\nu) \boldsymbol{\varepsilon}(\nabla w)+\nu \operatorname{div} \nabla w \boldsymbol{I}),
$$

which implies that the shear force $\boldsymbol{Q}$ satisfies the equilibrium equation:

$$
\boldsymbol{Q}=-\operatorname{div} \boldsymbol{M} \quad \text { and } \quad-\operatorname{div} \boldsymbol{Q}=g .
$$

With these notation, and assuming that the load is sufficiently regular, the Kirchhoff plate bending problem can be written as the well known biharmonic problem: Find the deflection $w$ such that

$$
\begin{array}{ll}
\mathrm{D} \Delta^{2} w=g & \text { in } \Omega, \\
w=0, \quad \nabla w \cdot \boldsymbol{n}=0 & \text { on } \Gamma_{\mathrm{C}}, \\
w=0, \boldsymbol{n} \cdot \boldsymbol{M} \boldsymbol{n}=0 & \text { on } \Gamma_{\mathrm{S}}, \\
\boldsymbol{n} \cdot \boldsymbol{M} \boldsymbol{n}=0, \quad \frac{\partial}{\partial \boldsymbol{s}}(\boldsymbol{s} \cdot \boldsymbol{M} \boldsymbol{n})+\boldsymbol{n} \cdot \boldsymbol{d i v} \boldsymbol{M}=0 & \text { on } \Gamma_{\mathrm{F}}, \\
\left(\boldsymbol{s}_{1} \cdot \boldsymbol{M} \boldsymbol{n}_{1}\right)(c)=\left(\boldsymbol{s}_{2} \cdot \boldsymbol{M} \boldsymbol{n}_{2}\right)(c) & \forall c \in \mathcal{V},
\end{array}
$$

where $\boldsymbol{n}$ and $\boldsymbol{s}$ are, respectively, the unit outward normal and the unit counterclockwise tangent to the boundary. By the indices 1 and 2 we denote the sides of the boundary angle at a corner point $c$. 


\subsection{The scaled mixed formulation}

For our analysis, it is convenient to introduce the mixed formulation. First, we assume that the loading is scaled as $g=\mathrm{Gt}^{3} f$ with $f$ fixed. Then the problem (2.6) becomes independent of the plate thickness:

$$
\frac{1}{6(1-\nu)} \Delta^{2} w=f \quad \text { in } \Omega
$$

The corresponding scaled moment and shear force are, respectively,

$$
\boldsymbol{m}(\nabla w)=\frac{\boldsymbol{M}(\nabla w)}{\mathrm{G} t^{3}} \quad \text { and } \quad \boldsymbol{q}=\frac{\boldsymbol{Q}}{\mathrm{G} t^{3}} .
$$

In the mixed formulation, the rotation and the shear force, respectively, are taken as new unknowns:

$$
\boldsymbol{\beta}=\nabla w \quad \text { and } \quad \boldsymbol{q}=-\operatorname{div} \boldsymbol{m}(\boldsymbol{\beta})=-\boldsymbol{L} \boldsymbol{\beta},
$$

where we have introduced a partial differential operator $\boldsymbol{L}$. Now, the scaled mixed problem reads: find the deflection $w$, rotation $\boldsymbol{\beta}$ and the shear force $\boldsymbol{q}$ such that

$$
\begin{aligned}
-\operatorname{div} \boldsymbol{q} & =f, \\
\boldsymbol{L} \boldsymbol{\beta}+\boldsymbol{q} & =\mathbf{0}, \\
\nabla w-\boldsymbol{\beta} & =\mathbf{0}, \quad \text { in } \Omega,
\end{aligned}
$$

with the boundary conditions

$$
\begin{aligned}
w & =0, \boldsymbol{\beta}=\mathbf{0}, \quad \text { on } \Gamma_{\mathrm{C}}, \\
w=0, \boldsymbol{\beta} \cdot \boldsymbol{s} & =0, \boldsymbol{n} \cdot \boldsymbol{m}(\boldsymbol{\beta}) \boldsymbol{n}=0, \quad \text { on } \Gamma_{\mathrm{S}}, \\
\frac{\partial w}{\partial \boldsymbol{s}}-\boldsymbol{\beta} \cdot \boldsymbol{s}=0, \boldsymbol{n} \cdot \boldsymbol{m}(\boldsymbol{\beta}) \boldsymbol{n} & =0, \frac{\partial}{\partial \boldsymbol{s}}(\boldsymbol{s} \cdot \boldsymbol{m}(\boldsymbol{\beta}) \boldsymbol{n})-\boldsymbol{q} \cdot \boldsymbol{n}=0, \quad \text { on } \Gamma_{\mathrm{F}},
\end{aligned}
$$

and the corner conditions

$$
\left(\boldsymbol{s}_{1} \cdot \boldsymbol{m}(\boldsymbol{\beta}) \boldsymbol{n}_{1}\right)(c)=\left(\boldsymbol{s}_{2} \cdot \boldsymbol{m}(\boldsymbol{\beta}) \boldsymbol{n}_{2}\right)(c) \quad \forall c \in \mathcal{V}
$$

The function spaces for the kinematic unknowns $w$ and $\boldsymbol{\beta}$ are defined as

$$
\begin{aligned}
& W=\left\{v \in H^{1}(\Omega) \mid v=0 \text { on } \Gamma_{\mathrm{C}} \cup \Gamma_{\mathrm{S}}\right\} \\
& \boldsymbol{V}=\left\{\boldsymbol{\eta} \in\left[H^{1}(\Omega)\right]^{2} \mid \boldsymbol{\eta}=\mathbf{0} \text { on } \Gamma_{\mathrm{C}}, \boldsymbol{\eta} \cdot \boldsymbol{s}=0 \text { on } \Gamma_{\mathrm{S}}\right\} .
\end{aligned}
$$

\section{The finite element method}

In this section, we will introduce our finite element method. We start by presenting the approach introduced by Hughes and Franca [19] for ReissnerMindlin plates and apply it for the Kirchhoff model. Then, following our 
earlier papers $[25,20]$, we show how the method formulated in kinematic variables can be obtained.

Even if our method is stable for all choices of finite element spaces, we will, for simplicity, present it for the polynomial degrees that yield an optimal convergence rate. Hence, let a regular family of meshes on $\Omega$ be given. For the integer $k \geq 1$, we then define the discrete spaces

$$
\begin{aligned}
W_{h} & =\left\{w \in W \quad \mid w_{\mid K} \in R_{k+1}(K) \forall K \in \mathcal{C}_{h}\right\}, \\
\boldsymbol{V}_{h} & =\left\{\boldsymbol{\eta} \in \boldsymbol{V} \mid \boldsymbol{\eta}_{\mid K} \in\left[R_{k}(K)\right]^{2} \quad \forall K \in \mathcal{C}_{h}\right\}
\end{aligned}
$$

for the approximations of the deflection and the rotation, respectively. Here $\mathcal{C}_{h}$ represents the collection of all the elements $K$ of the mesh. The local polynomial spaces are defines as follows:

$$
R_{k}(K)= \begin{cases}P_{k}(K) & \text { for triangular } K \\ Q_{k}(K) & \text { for quadrilateral } K,\end{cases}
$$

where $P_{k}(K)$ is the space of polynomials of degree at most $k$ on a triangle $K$ and $Q_{k}(K)$ is the corresponding space for quadrangles. We note that mixing triangular and quadrilateral elements in the mesh is allowed.

In the sequel, we will indicate with $h_{K}$ the diameter of each element $K$, while $h$ will indicate the maximum size of all the elements in the mesh. Furthermore, we will indicate with $E$ a general edge of the triangulation and with $h_{E}$ the length of $E$. In the derivation of the method, we will use the auxiliar space

$$
\boldsymbol{Q}_{h}=\left\{\boldsymbol{r} \in\left[L^{2}(\Omega)\right]^{2} \mid \boldsymbol{r}_{\mid K} \in\left[R_{l}(K)\right]^{2} \forall K \in \mathcal{C}_{h}\right\}
$$

for some $l \geq k-1$. The integer value $l$ will be specified below.

To derive the method, in the framework of Hughes and Franca [19], we take the inner product of the partial differential equation (2.11) with a test function $\boldsymbol{\eta} \in \boldsymbol{V}_{h}$ and integrate by parts yielding

$$
a(\boldsymbol{\beta}, \boldsymbol{\eta})-\langle\boldsymbol{m}(\boldsymbol{\beta}) \boldsymbol{n}, \boldsymbol{\eta}\rangle_{\Gamma}-(\boldsymbol{q}, \boldsymbol{\eta})=0,
$$

where

$$
a(\boldsymbol{\beta}, \boldsymbol{\eta})=(\boldsymbol{m}(\boldsymbol{\beta}), \boldsymbol{\varepsilon}(\boldsymbol{\eta})) .
$$

Here and below, $\langle\cdot, \cdot\rangle_{\Gamma}$ denotes the $L^{2}$-inner product along the boundary $\Gamma$.

Using the boundary conditions (2.14) and (2.15), and the ones in (2.18) imposed on $\boldsymbol{\eta}$, we have

$$
\begin{aligned}
\langle\boldsymbol{m}(\boldsymbol{\beta}) \boldsymbol{n}, \boldsymbol{\eta}\rangle_{\Gamma} & =\langle\boldsymbol{n} \cdot \boldsymbol{m}(\boldsymbol{\beta}) \boldsymbol{n}, \boldsymbol{\eta} \cdot \boldsymbol{n}\rangle_{\Gamma}+\langle\boldsymbol{s} \cdot \boldsymbol{m}(\boldsymbol{\beta}) \boldsymbol{n}, \boldsymbol{\eta} \cdot \boldsymbol{s}\rangle_{\Gamma} \\
& =\langle\boldsymbol{s} \cdot \boldsymbol{m}(\boldsymbol{\beta}) \boldsymbol{n}, \boldsymbol{\eta} \cdot \boldsymbol{s}\rangle_{\Gamma_{\mathrm{F}}}
\end{aligned}
$$

Hence, it holds

$$
a(\boldsymbol{\beta}, \boldsymbol{\eta})-\langle\boldsymbol{s} \cdot \boldsymbol{m}(\boldsymbol{\beta}) \boldsymbol{n}, \boldsymbol{\eta} \cdot \boldsymbol{s}\rangle_{\Gamma_{\mathrm{F}}}-(\boldsymbol{q}, \boldsymbol{\eta})=0
$$


Next, we take the inner product of the equilibrium equation (2.10) with $v \in W_{h}$, integrate by parts, use the boundary conditions imposed in $W_{h} \subset W$ and the third boundary condition in (2.15), which gives

$$
\begin{aligned}
0 & =(\operatorname{div} \boldsymbol{q}+f, v)=(f, v)-(\boldsymbol{q}, \nabla v)+\langle\boldsymbol{q} \cdot \boldsymbol{n}, v\rangle_{\Gamma} \\
& =(f, v)-(\boldsymbol{q}, \nabla v)+\langle\boldsymbol{q} \cdot \boldsymbol{n}, v\rangle_{\Gamma_{\mathrm{F}}} \\
& =(f, v)-(\boldsymbol{q}, \nabla v)+\left\langle\frac{\partial}{\partial \boldsymbol{s}}(\boldsymbol{s} \cdot \boldsymbol{m}(\boldsymbol{\beta}) \boldsymbol{n}), v\right\rangle_{\Gamma_{\mathrm{F}}} .
\end{aligned}
$$

Next, the last part above is treated by integration by parts and using the corner conditions (2.16),

$$
\left\langle\frac{\partial}{\partial \boldsymbol{s}}(\boldsymbol{s} \cdot \boldsymbol{m}(\boldsymbol{\beta}) \boldsymbol{n}), v\right\rangle_{\Gamma_{\mathrm{F}}}=-\langle\boldsymbol{s} \cdot \boldsymbol{m}(\boldsymbol{\beta}) \boldsymbol{n}, \nabla v \cdot \boldsymbol{s}\rangle_{\Gamma_{\mathrm{F}}} .
$$

Inserting this in the preceding equation we get

$$
(\boldsymbol{q}, \nabla v)+\langle\boldsymbol{s} \cdot \boldsymbol{m}(\boldsymbol{\beta}) \boldsymbol{n}, \nabla v \cdot \boldsymbol{s}\rangle_{\Gamma_{\mathrm{F}}}=(f, v) .
$$

Now, combining (3.8) and (3.11) we see that the solution triple $(w, \boldsymbol{\beta}, \boldsymbol{q})$ satisfies

$$
a(\boldsymbol{\beta}, \boldsymbol{\eta})+(\boldsymbol{q}, \nabla v-\boldsymbol{\eta})+\langle\boldsymbol{s} \cdot \boldsymbol{m}(\boldsymbol{\beta}) \boldsymbol{n},[\nabla v-\boldsymbol{\eta}] \cdot \boldsymbol{s}\rangle_{\Gamma_{\mathrm{F}}}=(f, v)
$$

for all $(v, \boldsymbol{\eta}) \in W_{h} \times \boldsymbol{V}_{h}$. To symmetrize the formulation above, we add the relation

$$
(\nabla w-\boldsymbol{\beta}, \boldsymbol{r})=0
$$

for $\boldsymbol{r} \in \boldsymbol{Q}_{h}$, obtained from (2.12), and

$$
\langle[\nabla w-\boldsymbol{\beta}] \cdot \boldsymbol{s}, \boldsymbol{s} \cdot \boldsymbol{m}(\boldsymbol{\eta}) \boldsymbol{n}\rangle_{\Gamma_{\mathrm{F}}}=0,
$$

obtained from (2.15).

Combining (3.12)-(3.14) we now conclude that the solution triple $(w, \boldsymbol{\beta}, \boldsymbol{q})$ to $(2.11)-(2.16)$ satisfies

$$
\begin{aligned}
& a(\boldsymbol{\beta}, \boldsymbol{\eta})+(\boldsymbol{q}, \nabla v-\boldsymbol{\eta})+(\nabla w-\boldsymbol{\beta}, \boldsymbol{r}) \\
& \quad+\langle\boldsymbol{s} \cdot \boldsymbol{m}(\boldsymbol{\beta}) \boldsymbol{n},[\nabla v-\boldsymbol{\eta}] \cdot \boldsymbol{s}\rangle_{\Gamma_{\mathrm{F}}}+\langle[\nabla w-\boldsymbol{\beta}] \cdot \boldsymbol{s}, \boldsymbol{s} \cdot \boldsymbol{m}(\boldsymbol{\eta}) \boldsymbol{n}\rangle_{\Gamma_{\mathrm{F}}} \\
& =(f, v),
\end{aligned}
$$

for all $(v, \boldsymbol{\eta}, \boldsymbol{r}) \in W_{h} \times \boldsymbol{V}_{h} \times \boldsymbol{Q}_{h}$. This formulation we stabilize by adding the following "least-squares" terms

$$
\begin{array}{r}
-\alpha \sum_{K \in \mathcal{C}_{h}} h_{K}^{2}(\boldsymbol{L} \boldsymbol{\beta}+\boldsymbol{q}, \boldsymbol{L} \boldsymbol{\eta}+\boldsymbol{r})_{K}=0, \\
\gamma \sum_{E \in \mathcal{F}_{h}} h_{E}^{-1}\langle[\nabla w-\boldsymbol{\beta}] \cdot \boldsymbol{s},[\nabla v-\boldsymbol{\eta}] \cdot \boldsymbol{s}\rangle_{E}=0, \\
\mu \sum_{K \in \mathcal{C}_{h}} h_{K}^{-2}(\nabla w-\boldsymbol{\beta}, \nabla v-\boldsymbol{\eta})_{K}=0,
\end{array}
$$


with $\alpha>0, \gamma>0, \mu>0$. Here $\mathcal{F}_{h}$ represents the collection of all the boundary edges on $\Gamma_{\mathrm{F}}$. These consistent terms are obtained from (2.11), (2.15) and (2.12), respectively.

Hence, we conclude that the exact solution satisfies

$$
\mathcal{M}_{h}(w, \boldsymbol{\beta}, \boldsymbol{q} ; v, \boldsymbol{\eta}, \boldsymbol{r})=(f, v) \quad \forall(v, \boldsymbol{\eta}, \boldsymbol{r}) \in W_{h} \times \boldsymbol{V}_{h} \times \boldsymbol{Q}_{h}
$$

with (now $(w, \boldsymbol{\beta}, \boldsymbol{q})$ are dummy variables)

$$
\begin{aligned}
\mathcal{M}_{h}(w, \boldsymbol{\beta}, \boldsymbol{q} ; v, \boldsymbol{\eta}, \boldsymbol{r})= & a(\boldsymbol{\beta}, \boldsymbol{\eta})+(\boldsymbol{q}, \nabla v-\boldsymbol{\eta})+(\nabla w-\boldsymbol{\beta}, \boldsymbol{r}) \\
& +\langle\boldsymbol{s} \cdot \boldsymbol{m}(\boldsymbol{\beta}) \boldsymbol{n},[\nabla v-\boldsymbol{\eta}] \cdot \boldsymbol{s}\rangle_{\Gamma_{\mathrm{F}}} \\
& +\langle[\nabla w-\boldsymbol{\beta}] \cdot \boldsymbol{s}, \boldsymbol{s} \cdot \boldsymbol{m}(\boldsymbol{\eta}) \boldsymbol{n}\rangle_{\Gamma_{\mathrm{F}}} \\
& -\alpha \sum_{K \in \mathcal{C}_{h}} h_{K}^{2}(\boldsymbol{L} \boldsymbol{\beta}+\boldsymbol{q}, \boldsymbol{L} \boldsymbol{\eta}+\boldsymbol{r})_{K} \\
& +\gamma \sum_{E \in \mathcal{F}_{h}} h_{E}^{-1}\langle[\nabla w-\boldsymbol{\beta}] \cdot \boldsymbol{s},[\nabla v-\boldsymbol{\eta}] \cdot \boldsymbol{s}\rangle_{E} \\
& +\mu \sum_{K \in \mathcal{C}_{h}} h_{K}^{-2}(\nabla w-\boldsymbol{\beta}, \nabla v-\boldsymbol{\eta})_{K} .
\end{aligned}
$$

The stabilized method in the spirit of Hughes and Franca [19] would then read:

Method 3.1. Find $\left(w_{h}, \boldsymbol{\beta}_{h}, \boldsymbol{q}_{h}\right) \in W_{h} \times \boldsymbol{V}_{h} \times \boldsymbol{Q}_{h}$ such that

$$
\mathcal{M}_{h}\left(w_{h}, \boldsymbol{\beta}_{h}, \boldsymbol{q}_{h} ; v, \boldsymbol{\eta}, \boldsymbol{r}\right)=(f, v) \quad \forall(v, \boldsymbol{\eta}, \boldsymbol{r}) \in W_{h} \times \boldsymbol{V}_{h} \times \boldsymbol{Q}_{h}
$$

It is quite easy to see that this formulation is stable if $0<\mu<1,0<$ $\alpha<C_{I} / 4$ and $\gamma>2 / C_{I}^{\prime}$, where $C_{I}$ and $C_{I}^{\prime}$ are constants in inverse estimates, see below.

Next, the shear force would be eliminated by local condensation and hence the first conclusion would be to choose the shear space as small as possible, but still having an optimal convergence rate. This would lead to the choice $l=k-1$.

There is, however, other alternatives that we will utilize. First, as observed in [20], it turns out that when choosing the spaces so that $\nabla W_{h} \subset \boldsymbol{Q}_{h}$, i.e., choosing $l \geq k$, then the " $\mu$-term" can be dropped without sacrificing stability. Furthermore, as in [20], we now note that when we further enlarge the shear space such that $\boldsymbol{V}_{h} \subset \boldsymbol{Q}_{h}$ (i.e., $l \geq k+1$ ), then the "local condensation" can be done analytically yielding

$$
\boldsymbol{q}_{h \mid K}=\frac{1}{\alpha h_{K}^{2}}\left(\nabla w_{h}-\boldsymbol{\beta}_{h}-\alpha h_{K}^{2} \boldsymbol{L} \boldsymbol{\beta}_{h}\right)_{\mid K} \forall K \in \mathcal{C}_{h}
$$

Now, substituting this into (3.21) gives our stabilized formulation of kinematic variables. 
Method 3.2. Find $\left(w_{h}, \boldsymbol{\beta}_{h}\right) \in W_{h} \times \boldsymbol{V}_{h}$ such that

$$
\mathcal{A}_{h}\left(w_{h}, \boldsymbol{\beta}_{h} ; v, \boldsymbol{\eta}\right)=(f, v) \quad \forall(v, \boldsymbol{\eta}) \in W_{h} \times \boldsymbol{V}_{h},
$$

where the bilinear form is defined as

$$
\mathcal{A}_{h}(z, \boldsymbol{\phi} ; v, \boldsymbol{\eta})=\mathcal{B}_{h}(z, \boldsymbol{\phi} ; v, \boldsymbol{\eta})+\mathcal{D}_{h}(z, \boldsymbol{\phi} ; v, \boldsymbol{\eta}),
$$

where

$$
\begin{aligned}
\mathcal{B}_{h}(z, \boldsymbol{\phi} ; v, \boldsymbol{\eta}) & \\
= & a(\boldsymbol{\phi}, \boldsymbol{\eta})-\sum_{K \in \mathcal{C}_{h}} \alpha h_{K}^{2}(\boldsymbol{L} \boldsymbol{\phi}, \boldsymbol{L} \boldsymbol{\eta})_{K} \\
& +\sum_{K \in \mathcal{C}_{h}} \frac{1}{\alpha h_{K}^{2}}\left(\nabla z-\boldsymbol{\phi}-\alpha h_{K}^{2} \boldsymbol{L} \boldsymbol{\phi}, \nabla v-\boldsymbol{\eta}-\alpha h_{K}^{2} \boldsymbol{L} \boldsymbol{\eta}\right)_{K}
\end{aligned}
$$

with

$$
a(\boldsymbol{\phi}, \boldsymbol{\eta})=\frac{1}{6}\left((\varepsilon(\boldsymbol{\phi}), \boldsymbol{\varepsilon}(\boldsymbol{\eta}))+\frac{\nu}{1-\nu}(\operatorname{div} \boldsymbol{\phi}, \operatorname{div} \boldsymbol{\eta})\right)
$$

and

$$
\begin{aligned}
\mathcal{D}_{h}(z, \boldsymbol{\phi} ; v, \boldsymbol{\eta})= & \left\langle m_{n s}(\boldsymbol{\phi}),[\nabla v-\boldsymbol{\eta}] \cdot \boldsymbol{s}\right\rangle_{\Gamma_{\mathrm{F}}}+\left\langle[\nabla z-\boldsymbol{\phi}] \cdot \boldsymbol{s}, m_{n s}(\boldsymbol{\eta})\right\rangle_{\Gamma_{\mathrm{F}}} \\
& +\sum_{E \in \mathcal{F}_{h}} \frac{\gamma}{h_{E}}\langle[\nabla z-\boldsymbol{\phi}] \cdot \boldsymbol{s},[\nabla v-\boldsymbol{\eta}] \cdot \boldsymbol{s}\rangle_{E}
\end{aligned}
$$

for all $(z, \phi),(v, \boldsymbol{\eta}) \in W_{h} \times \boldsymbol{V}_{h}$, with $m_{n s}=\boldsymbol{s} \cdot \boldsymbol{m n}$.

From the way we have arrived at our formulation, it is clear that it is consistent.

Theorem 3.1. The exact solution $(w, \boldsymbol{\beta}) \in W \times \boldsymbol{V}$ satisfies

$$
\mathcal{A}_{h}(w, \boldsymbol{\beta} ; v, \boldsymbol{\eta})=(f, v) \quad \forall(v, \boldsymbol{\eta}) \in W_{h} \times \boldsymbol{V}_{h} .
$$

The consistency can be verified directly from the final formulation above as well, cf. [6].

Remark 3.1. We have split the discrete bilinear form as $\mathcal{A}_{h}=\mathcal{B}_{h}+\mathcal{D}_{h}$. Here $\mathcal{B}_{h}$ is the one obtained from the formulation of [25] by formally setting the (scaled) thickness $t=0$. This would yield an inconsistent method with a convergence rate $\mathcal{O}\left(h^{1 / 2}\right)$, cf. [6]. The first term in $\mathcal{D}_{h}$ yields the method consistent. The other two terms are for achieving the symmetry and stability.

Remark 3.2. Although the polynomial orders for the deflection and the rotation are different, the method can be easily implemented in a software framework providing hierarchial shape functions. Furthermore, implementing the lowest order element with $k=1$ is straightforward even in a framework that provides only the standard nodal shape functions of equal order for different variables. Moreover, in this case, it holds that $\boldsymbol{L} \boldsymbol{\eta}_{\mid K}=\mathbf{0}$ for all $K \in \mathcal{C}_{h}, \boldsymbol{\eta} \in \boldsymbol{V}_{h}$, and we have

$$
\mathcal{B}_{h}(z, \boldsymbol{\phi} ; v, \boldsymbol{\eta})=a(\boldsymbol{\phi}, \boldsymbol{\eta})+\sum_{K \in \mathcal{C}_{h}} \frac{1}{\alpha h_{K}^{2}}(\nabla z-\boldsymbol{\phi}, \nabla v-\boldsymbol{\eta})_{K} .
$$

Now, no upper limit has to be imposed on $\alpha$. 


\section{$4 \quad$ Stability and a-priori error estimates}

Here we show that our method is optimally convergent. For the deflection and the rotation $(v, \boldsymbol{\eta}) \in W_{h} \times \boldsymbol{V}_{h}$, we introduce the following mesh dependent norms:

$$
\begin{aligned}
& |(v, \boldsymbol{\eta})|_{h}^{2}=\sum_{K \in \mathcal{C}_{h}} h_{K}^{-2}\|\nabla v-\boldsymbol{\eta}\|_{0, K}^{2} \\
& \|v\|_{2, h}^{2}=\|v\|_{1}^{2}+\sum_{K \in \mathcal{C}_{h}}|v|_{2, K}^{2}+\sum_{E \in \mathcal{I}_{h}} h_{E}^{-1}\left\|\llbracket \frac{\partial v}{\partial \boldsymbol{n}} \rrbracket\right\|_{0, E}^{2}+\sum_{E \subset \Gamma_{\mathrm{C}}} h_{E}^{-1}\left\|\frac{\partial v}{\partial \boldsymbol{n}}\right\|_{0, E}^{2} \\
& \left\|\left.(v, \boldsymbol{\eta})\left|\left\|_{h}=\right\| \boldsymbol{\eta}\left\|_{1}+\right\| v \|_{2, h}+\right|(v, \boldsymbol{\eta})\right|_{h}\right.
\end{aligned}
$$

where $\mathcal{I}_{h}$ represents the collection of all the edges of the triangulation lying in the interior of the domain $\Omega$. Here $\llbracket \frac{\partial v}{\partial \boldsymbol{n}} \rrbracket_{\mid E}$ denotes the jump of $\frac{\partial v}{\partial \boldsymbol{n}}$ across the edge $E$.

For the error estimation of the deflection and the rotation, we use a practical alternative to the norm (4.3) in the computations of Section 6 as well as in the stability proof below. This auxiliar norm is equivalent to the norm ||$\cdot|\||_{h}$ :

Lemma 4.1. There exists a positive constant $C$ such that

$$
C\left|\|(v, \boldsymbol{\eta})\|\left\|_{h} \leq\right\| \boldsymbol{\eta}\left\|_{1}+|(v, \boldsymbol{\eta})|_{h} \leq\right\|\right||(v, \boldsymbol{\eta})| \|_{h} \quad \forall(v, \boldsymbol{\eta}) \in W_{h} \times \boldsymbol{V}_{h} .
$$

For the a-priori error estimate, we first prove the stability of the method. Before this, we state the following two inverse inequalities which trivially follow from classical scaling arguments and the coercivity of the bilinear form $a$.

Lemma 4.2. Given any triangulation $\mathcal{C}_{h}$, there exist positive constants $C_{I}$ and $C_{I}^{\prime}$ such that,

$$
\begin{aligned}
& C_{I} \sum_{K \in \mathcal{C}_{h}} h_{K}^{2}\|\boldsymbol{L} \boldsymbol{\phi}\|_{0, K}^{2} \leq a(\boldsymbol{\phi}, \boldsymbol{\phi}) \quad \forall \boldsymbol{\phi} \in \boldsymbol{V}_{h}, \\
& C_{I}^{\prime} \sum_{E \in \mathcal{F}_{h}} h_{E}\left\|m_{n s}(\boldsymbol{\phi})\right\|_{0, E}^{2} \leq a(\boldsymbol{\phi}, \boldsymbol{\phi}) \quad \forall \boldsymbol{\phi} \in \boldsymbol{V}_{h} .
\end{aligned}
$$

As the stability is the crucial property of the method, we repeat the proof below. We want to stress that the proof is independent of the choice of the local polynomial spaces.

Theorem 4.1. Let $0<\alpha<C_{I} / 4$ and $\gamma>2 / C_{I}^{\prime}$. Then there exists a positive constant $C$ such that

$$
\mathcal{A}_{h}(v, \boldsymbol{\eta} ; v, \boldsymbol{\eta}) \geq C\|\|(v, \boldsymbol{\eta}) \|_{h}^{2} \quad \forall(v, \boldsymbol{\eta}) \in W_{h} \times \boldsymbol{V}_{h}
$$


Proof. First, the definition (3.25) for the bilinear form and the first inverse estimate of Lemma 4.2 gives

$$
\begin{aligned}
& \mathcal{B}_{h}(v, \boldsymbol{\eta} ; v, \boldsymbol{\eta}) \\
& \quad=a(\boldsymbol{\eta}, \boldsymbol{\eta})-\sum_{K \in \mathcal{C}_{h}} \alpha h_{K}^{2}\|\boldsymbol{L} \boldsymbol{\eta}\|_{0, K}^{2}+\sum_{K \in \mathcal{C}_{h}} \frac{1}{\alpha h_{K}^{2}}\left\|\nabla v-\boldsymbol{\eta}-\alpha h_{K}^{2} \boldsymbol{L} \boldsymbol{\eta}\right\|_{0, K}^{2} \\
& \quad \geq\left(1-\frac{\alpha}{C_{I}}\right) a(\boldsymbol{\eta}, \boldsymbol{\eta})+\sum_{K \in \mathcal{C}_{h}} \frac{1}{\alpha h_{K}^{2}}\left\|\nabla v-\boldsymbol{\eta}-\alpha h_{K}^{2} \boldsymbol{L} \boldsymbol{\eta}\right\|_{0, K}^{2} .
\end{aligned}
$$

Next, by using the definition (3.27), Cauchy's and Young's inequalities, and the second inverse inequality of Lemma 4.2 , we obtain

$$
\begin{aligned}
& \mathcal{D}_{h}(v, \boldsymbol{\eta} ; v, \boldsymbol{\eta})=\sum_{E \in \mathcal{F}_{h}}\left(2\left(m_{n s}(\boldsymbol{\eta}),[\nabla v-\boldsymbol{\eta}] \cdot \boldsymbol{s}\right)_{E}+\frac{\gamma}{h_{E}}\|[\nabla v-\boldsymbol{\eta}] \cdot \boldsymbol{s}\|_{0, E}^{2}\right) \\
& \geq \sum_{E \in \mathcal{F}_{h}}\left(-\frac{\gamma}{h_{E}}\|[\nabla v-\boldsymbol{\eta}] \cdot \boldsymbol{s}\|_{0, E}^{2}-\gamma^{-1} h_{E}\left\|m_{n s}(\boldsymbol{\eta})\right\|_{0, E}^{2}\right. \\
& \left.\quad+\frac{\gamma}{h_{E}}\|[\nabla v-\boldsymbol{\eta}] \cdot \boldsymbol{s}\|_{0, E}^{2}\right) \\
& =-\sum_{E \in \mathcal{F}_{h}} \gamma^{-1} h_{E}\left\|m_{n s}(\boldsymbol{\eta})\right\|_{0, E}^{2} \\
& \geq-\frac{1}{\gamma C_{I}^{\prime}} a(\boldsymbol{\eta}, \boldsymbol{\eta}) \geq-\frac{1}{2} a(\boldsymbol{\eta}, \boldsymbol{\eta}) .
\end{aligned}
$$

Joining (4.8) with (4.9) and using Korn's inequality leads to

$$
\begin{aligned}
& \mathcal{B}_{h}(v, \boldsymbol{\eta} ; v, \boldsymbol{\eta})+\mathcal{D}_{h}(v, \boldsymbol{\eta} ; v, \boldsymbol{\eta}) \\
& \quad \geq\left(\frac{1}{2}-\frac{\alpha}{C_{I}}\right) a(\boldsymbol{\eta}, \boldsymbol{\eta})+\sum_{K \in \mathcal{C}_{h}} \frac{1}{\alpha h_{K}^{2}}\left\|\nabla v-\boldsymbol{\eta}-\alpha h_{K}^{2} \boldsymbol{L} \boldsymbol{\eta}\right\|_{0, K}^{2} \\
& \quad \geq C\left(\|\boldsymbol{\eta}\|_{1}^{2}+\sum_{K \in \mathcal{C}_{h}} \frac{1}{\alpha h_{K}^{2}}\left\|\nabla v-\boldsymbol{\eta}-\alpha h_{K}^{2} \boldsymbol{L} \boldsymbol{\eta}\right\|_{0, K}^{2}\right) .
\end{aligned}
$$

From the triangle inequality, the first inverse estimate of Lemma 4.2, and the boundedness of the bilinear form $a$, it follows

$$
\begin{aligned}
& \sum_{K \in \mathcal{C}_{h}} \frac{1}{\alpha h_{K}^{2}}\|\nabla v-\boldsymbol{\eta}\|_{0, K}^{2} \\
& \quad \leq 2\left(\sum_{K \in \mathcal{C}_{h}} \frac{1}{\alpha h_{K}^{2}}\left\|\nabla v-\boldsymbol{\eta}-\alpha h_{K}^{2} \boldsymbol{L} \boldsymbol{\eta}\right\|_{0, K}^{2}+\sum_{K \in \mathcal{C}_{h}} \frac{1}{\alpha h_{K}^{2}}\left\|\alpha h_{K}^{2} \boldsymbol{L} \boldsymbol{\eta}\right\|_{0, K}^{2}\right) \\
& \quad \leq 2\left(\sum_{K \in \mathcal{C}_{h}} \frac{1}{\alpha h_{K}^{2}}\left\|\nabla v-\boldsymbol{\eta}-\alpha h_{K}^{2} \boldsymbol{L} \boldsymbol{\eta}\right\|_{0, K}^{2}+\sum_{K \in \mathcal{C}_{h}} \alpha h_{K}^{2}\|\boldsymbol{L} \boldsymbol{\eta}\|_{0, K}^{2}\right) \\
& \quad \leq C\left(\sum_{K \in \mathcal{C}_{h}} \frac{1}{\alpha h_{K}^{2}}\left\|\nabla v-\boldsymbol{\eta}-\alpha h_{K}^{2} \boldsymbol{L} \boldsymbol{\eta}\right\|_{0, K}^{2}+a(\boldsymbol{\eta}, \boldsymbol{\eta})\right) \\
& \quad \leq C\left(\sum_{K \in \mathcal{C}_{h}} \frac{1}{\alpha h_{K}^{2}}\left\|\nabla v-\boldsymbol{\eta}-\alpha h_{K}^{2} \boldsymbol{L} \boldsymbol{\eta}\right\|_{0, K}^{2}+\|\boldsymbol{\eta}\|_{1}^{2}\right),
\end{aligned}
$$


which, combined with (4.1) and (4.10), gives

$$
\mathcal{A}_{h}(v, \boldsymbol{\eta} ; v, \boldsymbol{\eta}) \geq C\left(\|\boldsymbol{\eta}\|_{1}^{2}+|(v, \boldsymbol{\eta})|_{h}^{2}\right) .
$$

The result then follows from the norm equivalenve of Lemma 4.1.

We then have the following a-priori error estimate, which directly follows from the stability, consistency and the interpolation estimates, see [6].

Theorem 4.2. Suppose that $0<\alpha<C_{I} / 4$ and $\gamma>2 / C_{I}^{\prime}$, and that the exact solution satisfies $w \in H^{s+2}(\Omega)$, with $1 \leq s \leq k$. Then it holds

$$
\left\|\left(w-w_{h}, \boldsymbol{\beta}-\boldsymbol{\beta}_{h}\right)\right\|_{h} \leq C h^{s}\|w\|_{s+2} .
$$

For the error analysis of the shear force, we note that the discrete shear force defined in (3.22) is a consistent approximation since it follows from (2.11) and (2.12) that the exact shear force satisfies

$$
\boldsymbol{q}_{\mid K}=\frac{1}{\alpha h_{K}^{2}}\left(\nabla w-\boldsymbol{\beta}-\alpha h_{K}^{2} \boldsymbol{L} \boldsymbol{\beta}\right)_{\mid K} \forall K \in \mathcal{C}_{h} .
$$

Now, the practical norm to use is the discrete negative norm defined as

$$
\|\boldsymbol{r}\|_{-1, h}=\left(\sum_{K \in \mathcal{C}_{h}} h_{K}^{2}\|\boldsymbol{r}\|_{0, K}^{2}\right)^{1 / 2} .
$$

With the assumption $w \in H^{s+2}(\Omega), s \geq 1$, it holds that $\boldsymbol{q} \in L^{2}(\Omega)$, and we then have the following estimate [6]:

Lemma 4.3. Suppose that $0<\alpha<C_{I} / 4$ and $\gamma>2 / C_{I}^{\prime}$, and that the exact solution satisfies $w \in H^{s+2}(\Omega)$, with $1 \leq s \leq k$. Then it holds

$$
\left\|\boldsymbol{q}-\boldsymbol{q}_{h}\right\|_{-1, h} \leq C h^{s}\|w\|_{s+2} .
$$

For an estimate in a non-discrete dual norm, we introduce the following notation: with the space

$$
\boldsymbol{V}_{*}=\left\{\boldsymbol{\eta} \in\left[H^{1}(\Omega)\right]^{2} \mid \boldsymbol{\eta}=\mathbf{0} \text { on } \Gamma_{\mathrm{C}}, \boldsymbol{\eta} \cdot \boldsymbol{s}=0 \text { on } \Gamma_{\mathrm{F}} \cup \Gamma_{\mathrm{S}}\right\},
$$

we define the dual norm as

$$
\|\boldsymbol{r}\|_{-1, *}=\sup _{\boldsymbol{\eta} \in \boldsymbol{V}_{*}} \frac{\langle\boldsymbol{r}, \boldsymbol{\eta}\rangle}{\|\boldsymbol{\eta}\|_{1}}
$$

Now, the corresponding error estimate is the following [6]:

Lemma 4.4. It holds

$$
\left\|\boldsymbol{q}-\boldsymbol{q}_{h}\right\|_{-1, *} \leq C h^{s}\|w\|_{s+2} .
$$

This dual norm and its local counterpart will be used in the a-posteriori error bounds below. For further discussion on a-priori error bounds and regularity, we refer to [6]. 


\section{A-posteriori error estimates}

We now briefly recall the reliability and efficiency results for the a-posteriori error estimator presented in [6]. To this end, we introduce

$$
\begin{aligned}
& \tilde{\eta}_{K}^{2}:=h_{K}^{4}\left\|f+\operatorname{div} \boldsymbol{q}_{h}\right\|_{0, K}^{2}+h_{K}^{-2}\left\|\nabla w_{h}-\boldsymbol{\beta}_{h}\right\|_{0, K}^{2}, \\
& \eta_{E}^{2}:=h_{E}^{3}\left\|\llbracket \boldsymbol{q}_{h} \cdot \boldsymbol{n} \rrbracket\right\|_{0, E}^{2}+h_{E}\left\|\llbracket \boldsymbol{m}\left(\boldsymbol{\beta}_{h}\right) \boldsymbol{n} \rrbracket\right\|_{0, E}^{2}, \\
& \eta_{S, E}^{2}:=h_{E}\left\|m_{n n}\left(\boldsymbol{\beta}_{h}\right)\right\|_{0, E}^{2}, \\
& \eta_{F, E}^{2}:=h_{E}\left\|m_{n n}\left(\boldsymbol{\beta}_{h}\right)\right\|_{0, E}^{2}+h_{E}^{3}\left\|\frac{\partial}{\partial s} m_{n s}\left(\boldsymbol{\beta}_{h}\right)-\boldsymbol{q}_{h} \cdot \boldsymbol{n}\right\|_{0, E}^{2},
\end{aligned}
$$

where $m_{n s}=\boldsymbol{s} \cdot \boldsymbol{m n}, m_{n n}=\boldsymbol{n} \cdot \boldsymbol{m n}$ and $\llbracket \cdot \rrbracket$ represents the jump operator (which is assumed to be equal to the function value on boundary edges). Then, for any element $K \in \mathcal{C}_{h}$, the local error indicator is defined as

$$
\eta_{K}:=\left(\tilde{\eta}_{K}^{2}+\frac{1}{2} \sum_{E \in I(K)} \eta_{E}^{2}+\sum_{E \in S(K)} \eta_{S, E}^{2}+\sum_{E \in F(K)} \eta_{F, E}^{2}\right)^{1 / 2}
$$

where $I(K)$ denotes the edges of the element $K$ lying in the interior of $\Omega$, while $S(K)$ and $F(K)$ represent the edges of $K$ on $\Gamma_{\mathrm{S}}$ and on $\Gamma_{\mathrm{F}}$, respectively. Finally, the global error indicator is defined as

$$
\eta:=\left(\sum_{K \in \mathcal{C}_{h}} \eta_{K}^{2}\right)^{1 / 2}
$$

For the error analysis in [6], we have assumed that a classical saturation assumption holds. We then have the following efficiency and reliability results for the error estimator.

Theorem 5.1. Let $0<\alpha<C_{I} / 4$ and $\gamma>2 / C_{I}^{\prime}$. Then there exists a positive constant $C$ such that

$$
\left\|\left(w-w_{h}, \boldsymbol{\beta}-\boldsymbol{\beta}_{h}\right)\right\|_{h}+\left\|\boldsymbol{q}-\boldsymbol{q}_{h}\right\|_{-1, *} \leq C \eta .
$$

For the lower bound, we define a local counterpart of the negative norm defined in (4.18) for the shear force:

$$
\|\boldsymbol{r}\|_{-1, *, \omega_{K}}=\sup _{\substack{\boldsymbol{\eta} \in V_{*} \\ \boldsymbol{\eta}=\mathbf{0} \text { in } \Omega \backslash \omega_{K}}} \frac{\langle\boldsymbol{r}, \boldsymbol{\eta}\rangle}{\|\boldsymbol{\eta}\|_{1}} .
$$

We then have the following reliability result:

Theorem 5.2. Let $0<\alpha<C_{I} / 4$ and $\gamma>2 / C_{I}^{\prime}$, and let $\omega_{K}$ be the collection of all the elements sharing an edge with the element $K$. Then it holds

$$
\eta_{K} \leq C\left(\left\|||\left(w-w_{h}, \boldsymbol{\beta}-\boldsymbol{\beta}_{h}\right)\right\|_{h, \omega_{K}}+\left\|\boldsymbol{q}-\boldsymbol{q}_{h}\right\|_{-1, *, \omega_{K}}+h_{K}^{2}\left\|f-f_{h}\right\|_{0, \omega_{K}}\right),
$$

where $f_{h}$ is some approximation of the load $f$ and $\|\mid\| \cdot\|\|_{h, \omega_{K}},\|\cdot\|_{0, \omega_{K}}$ represent, respectively, the standard restrictions of the norms $\left|\|\cdot \mid\|_{h}\right.$ and $\|\cdot\|_{0}$ to the domain $\omega_{K}$.

The proofs of these results can be found in [6]. 


\section{$6 \quad$ Numerical results}

In this section, we consider various benchmark tests with different kinds of domains, boundary conditions and loadings. First, we show the convergence results concerning the a-priori error estimates for the new Kirchhoff method and the corresponding Reissner-Mindlin limit method, i.e., respectively, with and without the additional bilinear form $\mathcal{D}_{h}$ in (3.27) for free boundary edges. Second, we present the results for the a-posteriori error estimator of the new method for various benchmark problems.

\subsection{Constants in the computations}

In all the cases, the values $\mathrm{E}=1$ and $\nu=0.3$ have been used for the material constants. The stability constants we have chosen for the lowest order element with $k=1$ are $\alpha=0.1$ and $\gamma=100$.

For the degree $k=2$, we have used local stability constants inspired by the inverse inequalities of Lemma 4.2. For any element $K$, the local stability constant $\alpha_{K}$ can be defined by

$$
\frac{1}{\alpha_{K}}=\theta^{-1} \max _{\phi \in P_{k}(K), a_{K}(\boldsymbol{\phi}, \boldsymbol{\phi}) \neq 0} \frac{h_{K}^{2}\|\boldsymbol{L} \boldsymbol{\phi}\|_{0, K}^{2}}{a_{K}(\boldsymbol{\phi}, \boldsymbol{\phi})},
$$

where $a_{K}$ represents the form $a$ in (3.26) restricted to the element $K$, while for the parameter $\theta$ satisfying $0<\theta<1 / 4$, we have used the value $\theta=1 / 10$. Similarly, for an edge $E$ on the free boundary, a local constant $\gamma_{E}$ can be defined by

$$
\gamma_{E}=\delta \max _{\phi \in P_{k}(K), a_{K}(\boldsymbol{\phi}, \boldsymbol{\phi}) \neq 0} \frac{h_{E}\left\|m_{n s}(\boldsymbol{\phi})\right\|_{0, E}^{2}}{a_{K}(\boldsymbol{\phi}, \boldsymbol{\phi})}
$$

where for the parameter $\delta>2$, we have used the value $\delta=3$.

We emphasize that the method is not very sensitive with respect to the stability parameters. Instead of the local parameters defined above, a wide range of global parameters, satisfying the inequalities $\alpha<\alpha_{K}, \gamma>\gamma_{K}$ for all $K \in \mathcal{C}_{h}$, can be used in computations, see Lemma 4.2 and Theorem 4.2.

\subsection{An a-priori test}

We consider the following Kirchhoff bending problem of a semi-infinite plate. The midsurface and the boundary of the plate, respectively, are described by the sets

$$
\Omega=\left\{(x, y) \in \mathbb{R}^{2} \mid y>0\right\} \quad \text { and } \quad \Gamma=\left\{(x, y) \in \mathbb{R}^{2} \mid y=0\right\} .
$$

The plate is assumed to be free on the boundary $\Gamma$ and subjected to the transverse loading $f(x)=\cos x / G$. The exact $x$-periodic solution of this 
problem is given in [1]:

$$
\begin{aligned}
& w=\left(1 / A+b e^{-y}+d y e^{-y}\right) \cos x, \\
& \beta_{x}=\left(-1 / A-b e^{-y}-d y e^{-y}\right) \sin x, \\
& \beta_{y}=\left(-b e^{-y}+d(1-y) e^{-y}\right) \cos x,
\end{aligned}
$$

where $A=\mathrm{G} /(6(1-\nu))$. The coefficients $b$ and $d$, depending on $\mathrm{G}$ and $\nu$, are given in the reference [1] for different types of boundary conditions on $\Gamma$.

Due to the smoothness of the solution, from Theorem 4.2 and Lemma 4.1 it immediately follows the convergence rate

$$
\left\|\boldsymbol{\beta}-\boldsymbol{\beta}_{h}\right\|_{1}+\left|\left(w-w_{h}, \boldsymbol{\beta}-\boldsymbol{\beta}_{h}\right)\right|_{h}=\mathcal{O}\left(h^{k}\right) .
$$

On the contrary, according to the observations in $[6,5]$, the convergence rate for the Reissner-Mindlin limit method, without the additional bilinear form $\mathcal{D}_{h}$, should be of order $\mathcal{O}\left(h^{1 / 2}\right)$.

We discretize the domain $D=(0, \pi / 2) \times(0,3 \pi / 4)$ and set the symmetry conditions on the vertical boundaries $\{x=0,0 \leq y \leq 3 \pi / 4\}$ and $\{x=$ $\pi / 2,0 \leq y \leq 3 \pi / 4\}$, while on the upper horizontal boundary $\{y=3 \pi / 4,0 \leq$ $x \leq \pi / 2\}$ we use the non-homogeneus Dirichlet conditions adopting the exact solution as a reference. Some sample meshes are shown in Figure 1.

Let $D_{b}$ represent the boundary domain $[0, \pi / 2] \times[0, \pi / 4]$, see Figure 1 . First, in Figure 2 (left), we show the error convergence for the moment component $m_{n s}$ in the norm $L^{2}\left(D_{b}\right)$, for the polynomial degrees $k=1,2$. The dashed line represents the convergence graph for the Reissner-Mindlin limit method, i.e., without the correction $\mathcal{D}_{h}$ in (3.27), while the solid line refers to the new formulation, Method 3.2. As predicted by the theory, the convergence rate for the Reissner-Mindlin limit method is $\mathcal{O}\left(h^{1 / 2}\right)$, while the modified method follows the rate $\mathcal{O}\left(h^{k}\right)$. In Figure 2 (right), the error is measured in the norm of (6.5) which is equivalent to the norm $\||\cdot|\|_{h}$ (cf. Lemma 4.1). In this norm as well, the convergence rate for Method 3.2 is of the correct order $\mathcal{O}\left(h^{k}\right)$. For the Reissner-Mindlin limit method with $k=1$, only a slight deterioration of the convergence rate is visible, while the case $k=2$ again clearly follows the rate $\mathcal{O}\left(h^{1 / 2}\right)$.

\subsection{A-posteriori tests}

In this section, we illustrate the robustness of the local error estimator by means of convergence graphs and meshes obtained from adaptively and uniformly refined computations. We restrict ourselves to the case of the lowest order element with $k=1$, i.e., with a linear rotation and a quadratic deflection.

For the first three problems, with convex rectangular domains, the exact solution can be found in the form of a trigonometric-hyperbolic series which we have used as a reference solution. In these problems, we compare the behavior of the estimated and true error, reported as the effectivity index, i.e., the ratio between these two errors. With these problems, we show that 
the effectivity index remains on a certain almost constant level, uniformly in the mesh size.

We then consider a set of problems with nonconvex domains, for which the behavior of the estimated errors are reported alone, due to the lack of exact solutions. In these problems, we focus on the comparison between the estimated errors for uniform and adaptive refinements. These problems comprise different types of boundary conditions as well.

\subsubsection{The adaptive solution strategy}

For the adaptive computations, we have implemented Method 3.2 in the open-source finite element software Elmer [18]. For adaptivity, we have used the following strategy. In the beginning, we prescribe a coarse starting mesh. Then, after computing the approximate solution and the corresponding error estimators, a complete remeshing is done by using Delaunay triangulations. The refining-coarsening strategy is based on the local error indicators and on the assumption that the local error is of the form

$$
\eta_{K}=C_{K} h_{K}^{p_{K}}
$$

for some constants $C_{K}$ and $p_{K}$. The new mesh is then built with the aim of having the error uniformly distributed over the elements. This approach is usually called the error balancing strategy.

The stopping criteria for the adaptive process is either a given tolerance for the maximum local estimator or the number of refinement steps. Between two subsequent adaptive steps we have used the value 1.5 for the change of the relative local mesh density ratio. For example, a subdomain currently covered by six elements is after the next remeshing step covered by four to nine elements. For the element size, neither a maximum nor a minimum have been prescribed.

\subsubsection{Convex rectangular domains}

For the first three problems, we compare the behavior of the estimated and true error, finally reported as the effectivity index, i.e., the ratio between these two errors.

Rectangle with simply supported boundaries. We consider the simply supported rectangle $\Omega=(0,1) \times(-1,1)$ with the uniform loading $f=1$. The exact solution for the problem can be found by writing the load as a trigonometric series in which the constants $L_{x}$ and $L_{y}$ denote the side lenghts of the rectangle, in the $x$ - and $y$-direction, respectively. For the uniform load, it holds

$$
f=\sum_{i, j \in \mathbb{N}} \chi_{i, j} \sin \left(\frac{i \pi}{L_{x}} x\right) \sin \left(\frac{j \pi}{L_{y}} y\right), \quad \chi_{i, j}=\frac{16 f}{\pi^{2} i j}
$$


where the sum is only for odd indices $i, j$. After some calculation, we get for the solution $[27]$

$$
w=\sum_{i, j \in \mathbb{N}} \varpi_{i, j} \sin \left(\frac{i \pi}{L_{x}} x\right) \sin \left(\frac{j \pi}{L_{y}} y\right), \quad \varpi_{i, j}=\frac{6 \chi_{i, j}}{\pi^{4}(1-\nu)\left(\frac{i^{2}}{L_{x}^{2}}+\frac{j^{2}}{L_{y}^{2}}\right)^{2}} .
$$

In our tests, it has been enough to take into account the first ten terms from the series of the reference solution (6.8).

According to $[21,8]$, the critical corner regularity is now $w \in H^{3}(\Omega)$. This implies, by Theorem 4.2 and Lemma 4.1, the convergence rate $\mathcal{O}\left(h^{\sigma}\right)$ with $\sigma=\min \{1, k\}=1$. We note that for quasiuniform meshes, it holds that $h \sim N^{-1 / 2}$, where $N$ denotes the number of elements in the mesh.

The convergence graphs for the adaptively refined meshes are shown in Figure 4 (right). The two upper graphs (solid lines) represent, respectively, the global error estimator (asterisks) and the global true error (squares), while the lower ones (dashed lines) are, respectively, the maximum local estimator (asterisks) and the maximum of the local true errors (squares). For clarity, the convergence rate $\mathcal{O}(h)$ is indicated in the same figure as well (dashed line).

All the convergence curves are in agreement with the theoretical results. Moreover, we emphasize that, in this problem with simply supported boundaries, the behavior of the estimated error is almost identical with the true error, up to a multiplicative constant, cf. the effectivity index reported below. Finally, the deflection of the problem is illustrated in Figure 4 (left).

Rectangle with simply supported and free boundaries. Second, we consider the rectangle $\Omega=(0,1) \times(-1,1)$ with the simply supported left and right boundaries $\{x=0,-1 \leq y \leq 1\},\{x=1,-1 \leq y \leq 1\}$ and free bottom and top boundaries $\{y=-1,0 \leq x \leq 1\},\{y=1,0 \leq x \leq 1\}$. As above, the loading is constant, $f=1$. The exact solution for the problem can be found by writing the load as a trigonometric series in which the constant $L_{x}$ denotes the side length of the rectangle in the $x$-direction. For the uniform loading,

$$
f=\sum_{i \in \mathbb{N}} \chi_{i} \sin \left(\frac{i \pi}{L_{x}} x\right), \quad \chi_{i}=\frac{4 f}{\pi i},
$$

where the sum is only for odd indices $i$. Then, the solution is written in the form $w=w_{0}+w_{f}$, where $\Delta^{2} w_{0}=0$ and $\Delta^{2} w_{f}=6(1-\nu) f$. Now, we have

$$
w_{f}=\sum_{i \in \mathbb{N}} \varpi_{i} \sin \left(\frac{i \pi}{L_{x}} x\right), \quad \varpi_{i}=\frac{6(1-\nu) \chi_{i}}{\left(\frac{i \pi}{L_{x}}\right)^{4}},
$$

and

$$
w_{0}=\sum_{i \in \mathbb{N}}\left(\frac{L_{x}}{i \pi}\right)^{2}\left(\xi_{i} \cosh \left(\frac{i \pi}{L_{x}} y\right)+\varphi_{i} \frac{i \pi}{L_{x}} y \sinh \left(\frac{i \pi}{L_{x}} y\right)\right) \sin \left(\frac{i \pi}{L_{x}} x\right)
$$

The constants $\xi_{i}$ and $\varphi_{i}$ are determined by the boundary conditions on the free boundaries (for more details, see [27]). 
According to $[21,8]$, the regularity in the corners is again $w \in H^{3}(\Omega)$ which implies the convergence rate $\mathcal{O}\left(h^{\sigma}\right)=1$.

The convergence graphs for the adaptively refined meshes are plotted in Figure 5 (left), the two upper graphs (solid lines) are for the global errors, the lower ones (dashed lines) for the maximum local errors.

Again, the convergence rate of the global errors is the same as the theoretical value of this problem. We note that, in this problem, including free boundaries as well, the behavior of the estimated error is almost identical with the true error, up to a multiplicative constant, cf. the effectivity index below.

Square with clamped boundaries. Third, we consider the clamped square $\Omega=(-1,1) \times(-1,1)$ with the uniform loading $f=1$. In order to find the exact solution, we apply the following steps given with details in [27]. First, we take a solution $w_{S}$, in a form of a trigometric series, for the same problem but with simply supported boundaries. This gives us the corresponding rotation $\boldsymbol{\beta}_{\mathrm{S}}$. Then, enforcing boundary moments along the boundaries, we derive the corresponding particular deflection $w_{M}$ and the corresponding rotation $\boldsymbol{\beta}_{M}$. Finally, the rotation $\boldsymbol{\beta}=\boldsymbol{\beta}_{S}+\boldsymbol{\beta}_{M}$ has to vanish on the clamped boundaries, which gives the final form for the rotation, and for the deflection $w=w_{S}+w_{M}$ as well.

According to $[21,8,9]$, the regularity in the corners is now $w \in H^{4.74}(\Omega)$. This implies the convergence rate $\mathcal{O}\left(h^{\sigma}\right), \sigma=\min \{2.74, k\}=1$ for $k=1$.

The convergence graphs for the adaptively refined meshes are shown in Figure 5 (right), together with the convergence rate $\mathcal{O}(h)$. The same comments as in the two previous problems apply for this problem as well.

Effectivity index for the different problem types. The effectivity index for the adaptive error estimator, i.e., the ratio between the estimated and true error, is shown in Figure 3 for the previous test problems.

The reported steps, between 8 and 9335 elements, are taken from the adaptive refinements. As can be seen in the figure, the effectivity index lies between 0.4 and 1.2; the dashed line representing the value 1 . In all the test cases, the effectivity index first decreases (between 8 and 200 elements) but then remains in the range $0.4 \ldots 0.8$ (between 200 and 9335 elements). More precisely, in the problem with clamped boundaries (squares) the value for the effectivity index remains around 0.5 , while for the simply supported and free boundaries (circles, triangles) it stays around 0.7 after the first steps.

The numerical computations above show that the effectivity index remains on a certain almost constant level uniformly in the mesh size for all the different types of problems. We emphasize that although we have used here the practical norm of (6.5), the effectivity index with respect to the norm $\||\cdot|\|_{h}$ is different only up to a constant level, due to the equivalence of these two norms. These observations indicate that the error estimator can be used as an reliable and efficient error measure. 


\subsubsection{Non-convex domains}

In the sequel, we use the error estimator as the only error measure, due to the lack of exact solutions for the following benchmark problems. A similar approach has been applied for Reissner-Mindlin plates in [11, 12, 10].

L-shaped domain with simply supported boundaries. The first nonconvex problem is the L-shaped domain $\Omega$ with the corners $(0,0),(2,0)$, $(2,1),(1,1),(1,2)$ and $(0,2)$. The plate is uniformly loaded, $f=1$, and all the boundaries are simply supported.

According to $[21,8]$, the regularity in the critical L-corner is now $w \in$ $H^{7 / 3}(\Omega)$. This implies, by Theorem 4.2 and Lemma 4.1, the convergence rate $\mathcal{O}\left(h^{\sigma}\right), \sigma=\min \{1 / 3, k\}=1 / 3$.

The convergence graphs for the uniformly (circles) and adaptively (triangles) refined meshes are shown in Figure 6 (right). The two upper graphs (solid lines) represent the global error estimator, while the lower ones (dashed lines) indicate the maximum local estimator. Moreover, we show in the same figure the convergence rates $\mathcal{O}(h)$ and $\mathcal{O}\left(h^{1 / 3}\right)$ (dashed lines). For the lowest order element with $k=1$, the convergence rate would be of order $\mathcal{O}(h)$ if there was no corner singularity in the solution. Now, due to the singularity in the simply supported L-corner, the convergence rate should be of order $\mathcal{O}\left(h^{1 / 3}\right)$. Finally, two example meshes from the adaptive process are depicted in Figure 7 and the deflection is illustrated in Figure 6 (left).

First, we recall that for quasiuniform meshes, it holds that $h \sim N^{-1 / 2}$, with $N$ denoting the number of elements in the mesh. With the uniform refinements (circles), the convergence rate of the error estimator clearly follows the value $\mathcal{O}\left(h^{1 / 3}\right)$. This holds for both the global error estimator and the maximum local estimator. Differently, after the first adaptive steps, the method shows its robustness in finding the corner singularity of the solution and refining locally near the L-corner. This is seen in both the convergence graphs (triangles), and it is clear as well when looking at the meshes in Figure 7.

L-shaped domain with a free corner. In this case, the two edges forming the reentrant corner are free, while the remaining ones are simply supported.

According to $[21,8]$, the regularity in the corner is now $w \in H^{2.64}(\Omega)$. This implies the convergence rate $\mathcal{O}\left(h^{\sigma}\right), \sigma=\min \{0.64, k\}=0.64$.

The convergence graphs for the uniformly (circles) and adaptively (triangles) refined meshes are plotted in Figure 8 (right), together with the convergence rates $\mathcal{O}(h)$ and $\mathcal{O}\left(h^{0.64}\right)$ (dashed lines). Now, due to the singularity in the free L-corner, the convergence rate should be of order $\mathcal{O}\left(h^{0.64}\right)$. Two example meshes from the adaptive process are depicted in Figure 9. Finally, the deflection is illustrated in Figure 8 (left).

First, in the coarse mesh of Figure 9 (left), the error is concentrated near to the free boundaries. However, after some adaptive steps the error and refinements concentrate locally near to the L-corner, see Figure 9 (right).

L-shaped domain with a clamped corner. In this test problem, the 
two edges forming the reentrant corner are clamped, while the remaining ones are again simply supported.

According to $[21,8,9]$, the regularity in the corner is now $w \in H^{2.54}(\Omega)$ which implies the convergence rate $\mathcal{O}\left(h^{\sigma}\right)$ with $\sigma=0.54$.

The convergence graphs for the uniformly (circles) and adaptively (triangles) refined meshes are presented in Figure 10 (right), together with the convergence rates $\mathcal{O}(h)$ and $\mathcal{O}\left(h^{0.54}\right)$ (dashed lines). Now, due to the singularity in the clamped L-corner, the convergence rate should be of order $\mathcal{O}\left(h^{0.54}\right)$. Two example meshes from the adaptive process are depicted in Figure 11, and the deflection is illustrated in Figure 10 (left). The same comments as for the simply supported L-shaped domain above apply for the results of this problem as well.

K-shaped domain with clamped boundaries. To conclude the tests for the Kirchhoff-Love plate, we consider a uniformly loaded, $f=1, \mathrm{~K}$ shaped "corridor" domain with clamped boundaries. The corner points of the domain $\Omega$ are now $(0,0),(1,0),(1,1),(2,0),(2+\sqrt{2}, 0),(1.5,2),(2+\sqrt{2}, 4)$, $(2,4),(1,3),(1,4)$ and $(0,4)$.

According to $[21,8,9]$, the regularity in the straight L-corner is again $w \in$ $H^{2.54}(\Omega)$, while in the $\mathrm{V}$-corners $w \in H^{2.50}(\Omega)$. This implies by Theorem 4.2 and Lemma 4.1 the convergence rate $\mathcal{O}\left(h^{\sigma}\right), \sigma=\min \{0.54,0.50, k\}=0.50$.

The convergence graphs for the uniformly (circles) and adaptively (triangles) refined meshes are shown in Figure 12 (right), together with the convergence rates $\mathcal{O}(h), \mathcal{O}\left(h^{0.54}\right)$ and $\mathcal{O}\left(h^{0.50}\right)$ (dashed lines). For the lowest order element with $k=1$, due to the singularities in the $\mathrm{V}$-corners, the convergence rate should be of order $\mathcal{O}\left(h^{0.50}\right)$. Two example meshes from the adaptive process are shown in Figure 13 and the deflection is illustrated in Figure 12 (left).

In this case, there appears corner singularities of two different orders. After the first steps, the global convergence rate for the uniform refinements seems to follow the correct value $\mathcal{O}\left(h^{0.50}\right)$. Again, after the first adaptive steps, the method finds and clearly distinguishes all the separate corner singularities and refines locally near the L-corner and the two V-corners.

\section{Acknowledgements}

This work has been partly supported by the IMATI - CNR of Pavia and by the IHP Research Training Network Smart Systems; New Materials, Adaptive Systems and their Nonlinearities; Modeling, Control and Numerical Simulation. The computations of this work has been partly accomplished in the computing environment of CSC - the Finnish IT Center for Science.

\section{References}

[1] D. N. Arnold and R. S. Falk. Edge effects in the Reissner-Mindlin plate theory. In A. K. Noor, T. Belytschko, and J. C. Simo, editors, 
Analytic and Computational Models of Shells, pages 71-90, New York, 1989. ASME.

[2] D. N. Arnold, A. L. Madureira, and S. Zhang. On the range of applicability of the Reissner-Mindlin and Kirchhoff-Love plate bending models. J. Elasticity, 67:171-185, 2002.

[3] I. Babuška and B. Szabo. Finite Element Analysis. John Wiley \& Sons, Inc., New York, 1991.

[4] J.-L. Batoz, K.-J. Bathe, and L. W. Ho. A study of three-node triangular plate bending elements. Int. J. Num. Meths. Eng., 15:1771-1812, 1980.

[5] L. Beirão da Veiga. Finite element methods for a modified ReissnerMindlin free plate model. SIAM J. Num. Anal., 42:1572-1591, 2004.

[6] L. Beirão da Veiga, J. Niiranen, and R. Stenberg. A family of $C^{0}$ finite elements for Kirchhoff plates I: Error analysis. accepted for publication in SIAM J. Num. Anal. (preprint arXiv:math. NA/0611690).

[7] L. Beirão da Veiga, J. Niiranen, and R. Stenberg. A posteriori error estimates for the Morley plate bending element. Numer. Math., 106:165$179,2007$.

[8] H. Blum and R. Rannacher. On the boundary value problem of the biharmonic operator on domains with angular corners. Math. Meth. Apll. Sci., 2:556-581, 1980.

[9] J. H. Bramble, J. E. Pasciak, and C. Bacuta. Shift theorems for the biharmonic Dirichlet problem. In Recent Progress in Computational and Applied PDEs, pages 1-26, New York, 2002. Kluwer Academic/Plenum Publishers.

[10] C. Carstensen and J. Schöberl. Residual-based a posteriori error estimate for a mixed Reissner-Mindlin plate finite element method. Numer. Math., 103:225-250, 2006.

[11] C. Carstensen and K. Weinberg. Adaptive mixed finite element method for Reissner-Mindlin plate. Comp. Meths. Appl. Mech. Engrg., 190:6895-6908, 2001.

[12] C. Carstensen and K. Weinberg. An adaptive non-conforming finite element method for Reissner-Mindlin plates. Int. J. Num. Meths. Eng., 56:2313-2330, 2003.

[13] A. Charbonneau, K. Dossou, and R. Pierre. A residual-based a posteriori error estimator for the Ciarlet-Raviart formulation of the first biharmonic problem. Num. Meth. Part. Diff. Eq., 13:93-111, 1997.

[14] P. G. Ciarlet. The Finite Element Method for Elliptic Problems. NorthHolland, Amsterdam, 1987. 
[15] P. G. Ciarlet and P. Raviart. A mixed finite element method for the biharmonic equation. In C. de Boor, editor, Mathematical Aspects of Finite Elements in Partial Differential Equations, pages 125-145. Academic Press, New York, 1974.

[16] P. Destuynder and T. Nevers. Une modification du modèle de Mindlin pour les plaques minces en flexion présentant un bord libre. RAIRO Modél. Math. Anal. Numér., 22:217-242, 1988.

[17] G. Engel, K. Garikipati, T. J. R. Hughes, M. G. Larson, L. Matsei, and R. L. Taylor. Continuous/discontinuous finite element approximations of fourth-order elliptic problems in structural and continuum mechanics with applications to thin beams and plates, and strain gradient elasticity. Comp. Meths. Appl. Mech. Engrg., 191:3669-3750, 2002.

[18] Elmer finite element software homepage. http://www.csc.fi/elmer.

[19] T. J. R. Hughes and L. P. Franca. A mixed finite element formulation for Reissner-Mindlin plate theory: Uniform convergence of all higher-order spaces. Comp. Meths. Appl. Mech. Engrg., 67:223-240, 1988.

[20] M. Lyly and R. Stenberg. Stabilized finite element methods for Reissner-Mindlin plates. Forschungsbericht 4, Universität Innsbruck, Institut für Mathematik und Geometrie, Juli 1999. http://www.math.tkk.fi/ rstenber.

[21] H. Melzer and R. Rannacher. Spannungskonzentrationen in Eckpunkten der vertikal belasteten Kirchhoffschen Platte. Bauingenieur, 55:181-189, 1980 .

[22] W. Ming and J. Xu. The Morley element for fourth order elliptic equations in any dimensions. Numer. Math., 103:155-169, 2006.

[23] L. S. D. Morley. The triangular equilibrium element in the solution of plate bending problems. Aero. Quart., 19:149-169, 1968.

[24] J. Pitkäranta and M. Suri. Design principles and error analysis for reduced-shear plate-bending finite elements. Numer. Math., 75:223-266, 1996.

[25] R. Stenberg. A new finite element formulation for the plate bending problem. In P. Ciarlet, L. Trabucho, and J. M. Viano, editors, Asymptotic Methods for Elastic Structures, pages 209-221. Walter de Gruyter \& Co., Berlin - New York, 1995.

[26] R. Stenberg. On some techniques for approximating boundary conditions in the finite element method. J. Comp. Appl. Math., 63:139-148, 1995.

[27] S. Timoshenko and S. Woinowsky-Krieger. Theory of plates and shells. McGraw-Hill Book Company, Auckland, 1959. 

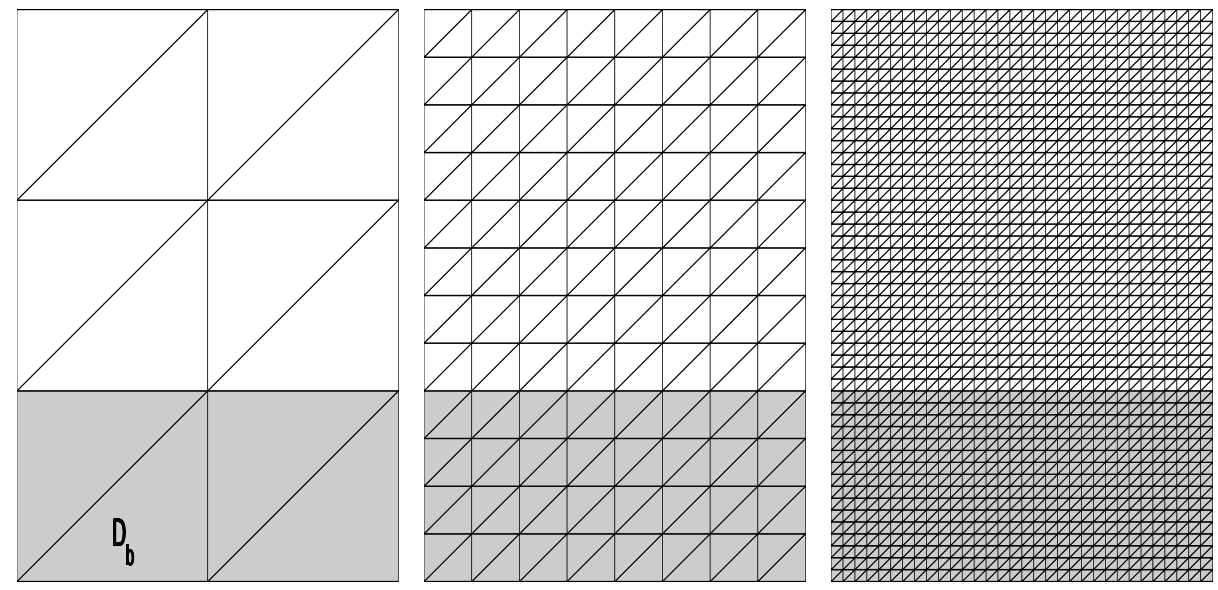

Figure 1: Samples of the adopted meshes and the boundary domain $D_{b}$.
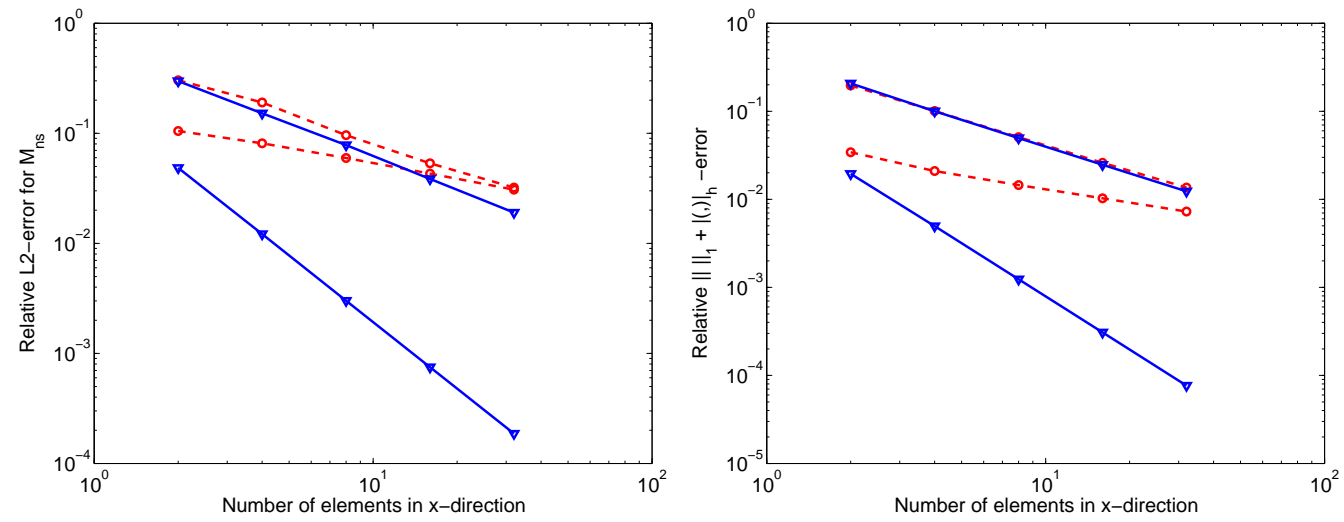

Figure 2: Free boundary: Convergence of the error for the moment component $m_{n s}(\boldsymbol{\beta})$ in the norm $L^{2}\left(D_{b}\right)$ with $k=1,2$; Convergence of the error in the norm $\left\|\boldsymbol{\beta}-\boldsymbol{\beta}_{h}\right\|_{1, D_{b}}+\left|\left(w-w_{h}, \boldsymbol{\beta}-\boldsymbol{\beta}_{h}\right)\right|_{h, D_{b}}$ with $k=1,2$; Dashed lines for the Reissner-Mindlin limit method, solid lines for the new method.

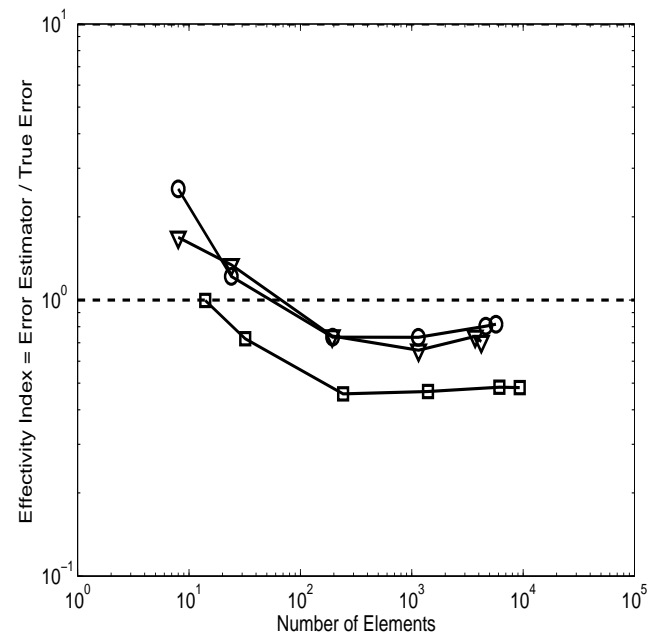

Figure 3: Effectivity index for the adaptive refinements: Clamped (squares), simply supported (circles), simply supported and free (triangles) boundaries. 

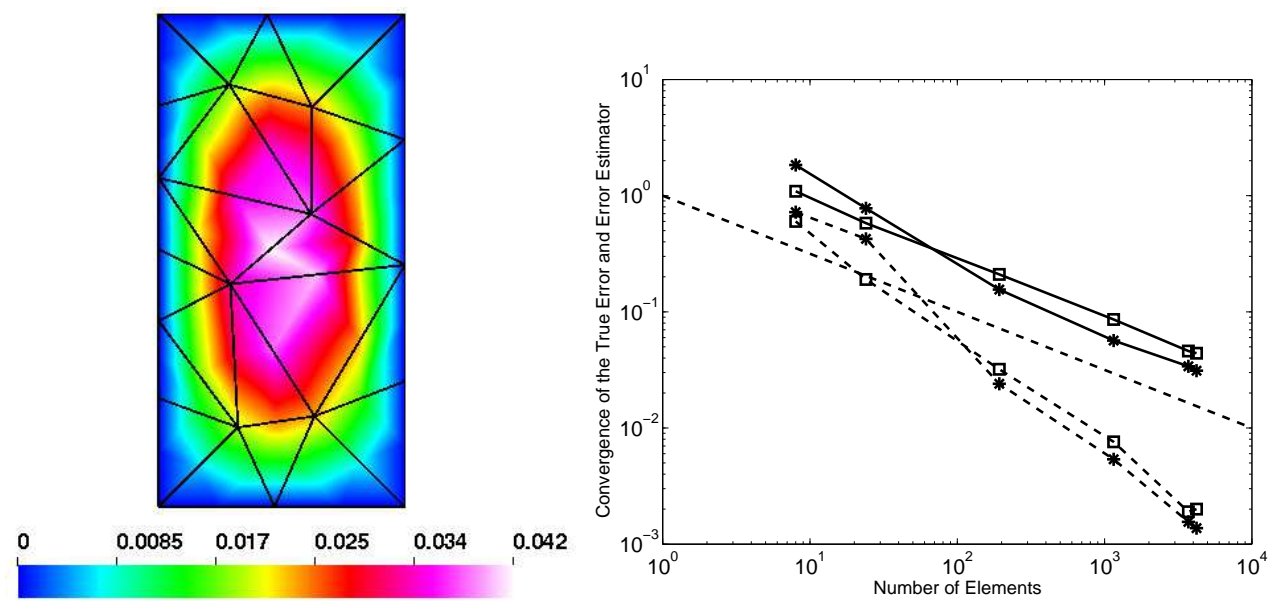

Figure 4: Simply supported boundary: Deflection distribution for the first mesh; Convergence of the true error (squares) and the error estimator (asterisks) for adaptive refinements; Solid lines for the global values, dashed lines for the local maximums.
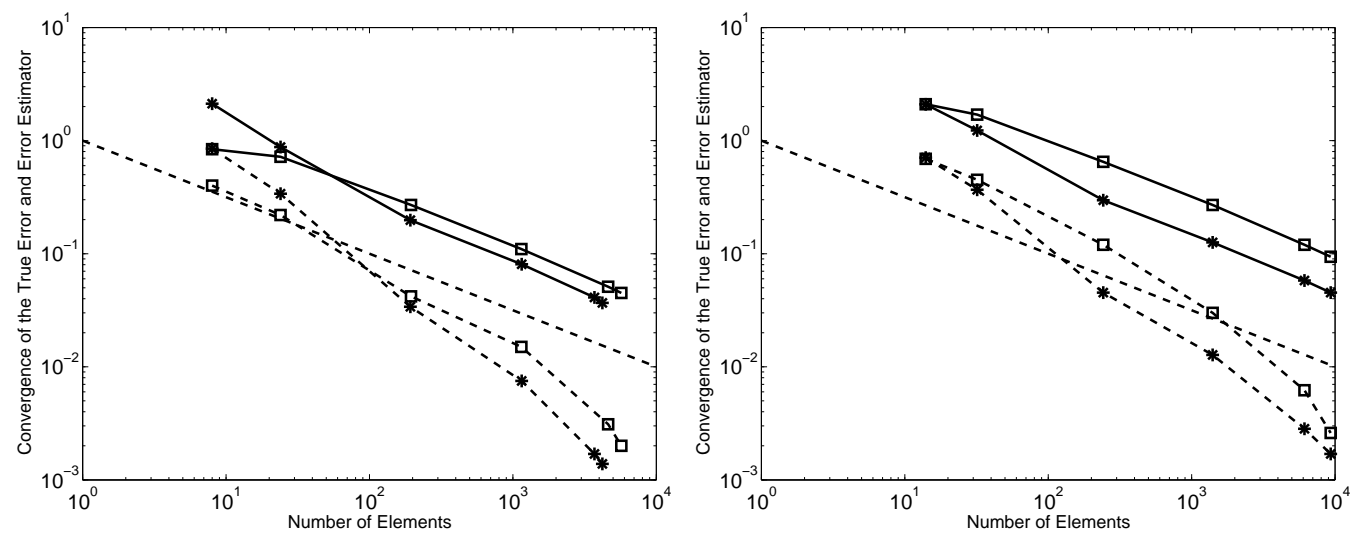

Figure 5: Simply supported and free boundary (left); Clamped boundary (right): Convergence of the true error (squares) and the error estimator (asterisks) for adaptive refinements; Solid lines for the global values, dashed lines for the local maximums. 

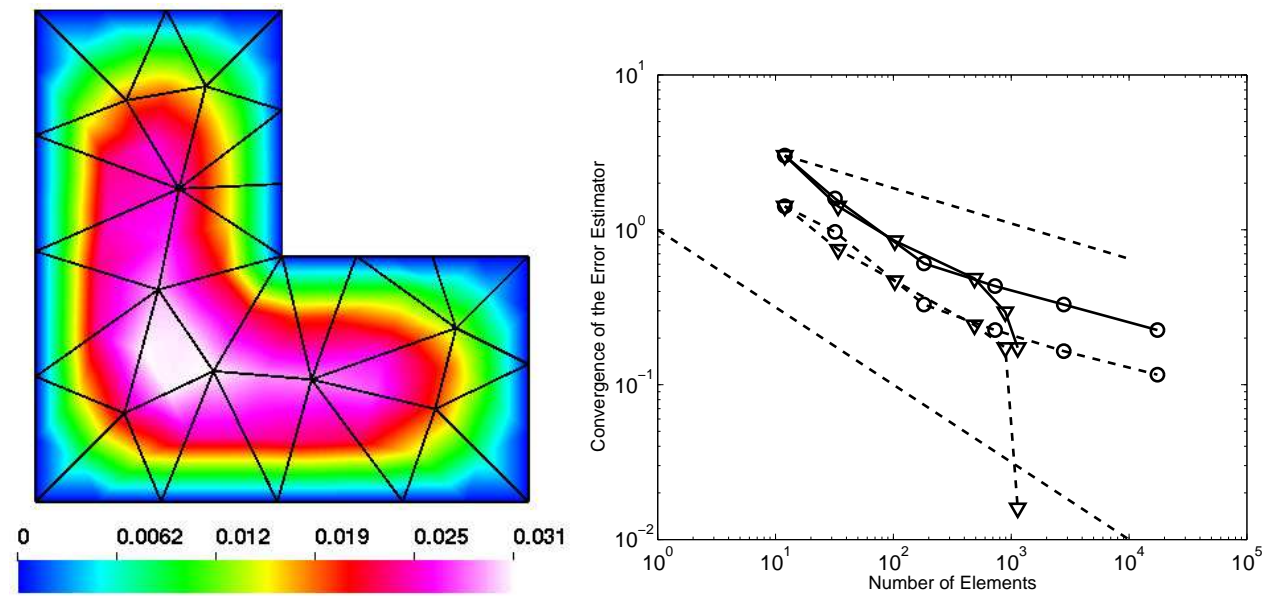

Figure 6: Simply supported L-corner: Deflection distribution for the first mesh; Convergence of the global estimator (solid lines) and the maximum local estimator (dashed lines); Circles for the uniform refinements, triangles for the adaptive refinements.
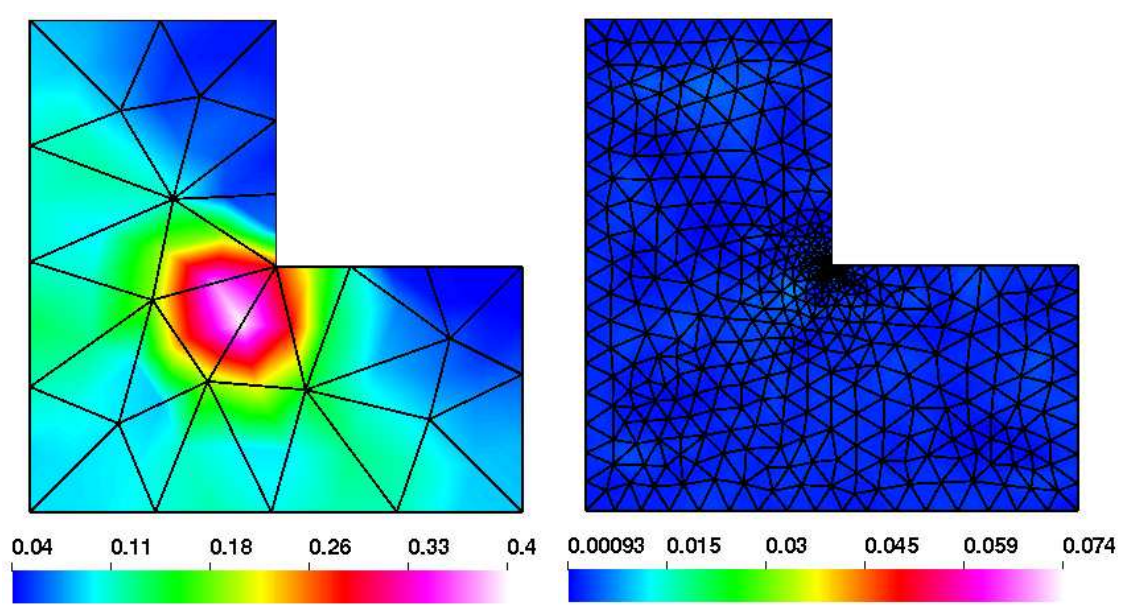

Figure 7: Simply supported L-corner: Distribution of the error estimator for two different refinement steps. 

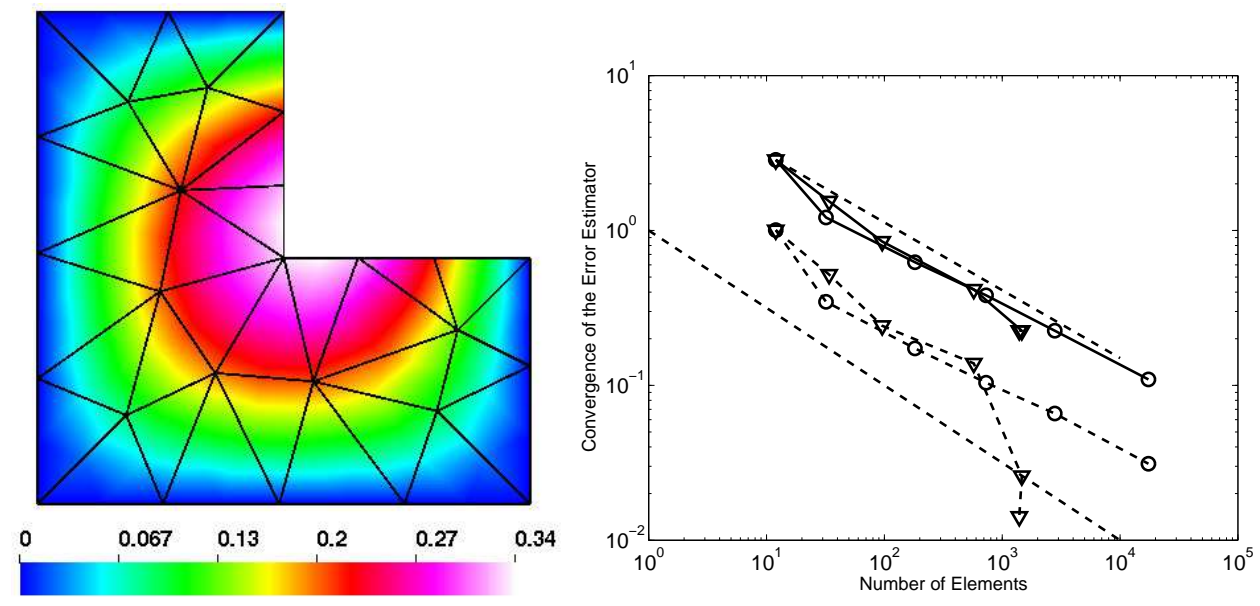

Figure 8: Free L-corner: Deflection distribution for the first mesh; Convergence of the global estimator (solid lines) and the maximum local estimator (dashed lines); Circles for the uniform refinements, triangles for the adaptive refinements.
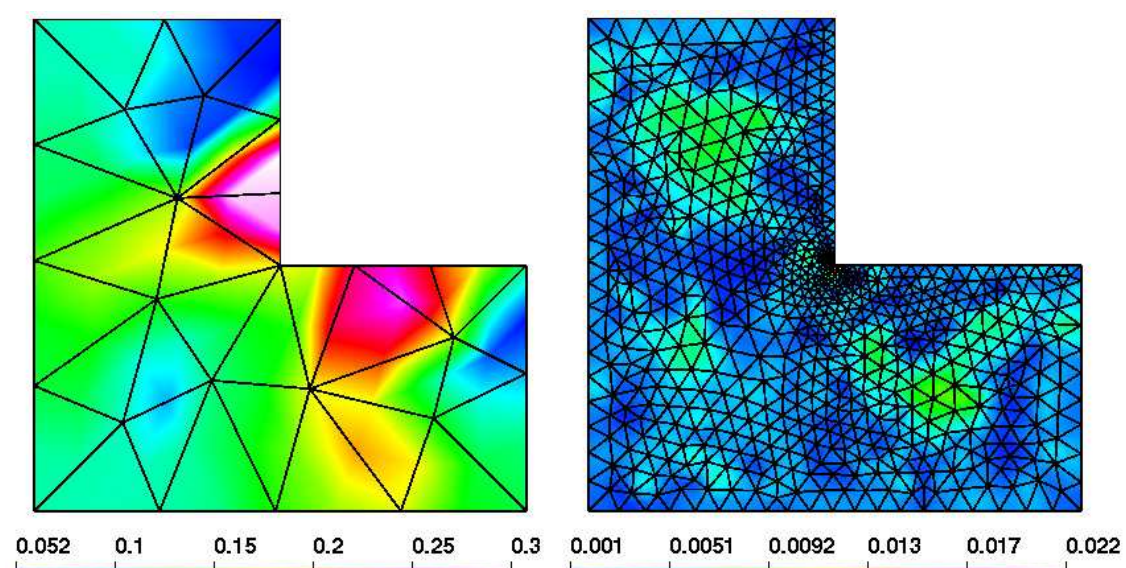

Figure 9: Free L-corner: Distribution of the error estimator for two different refinement steps. 

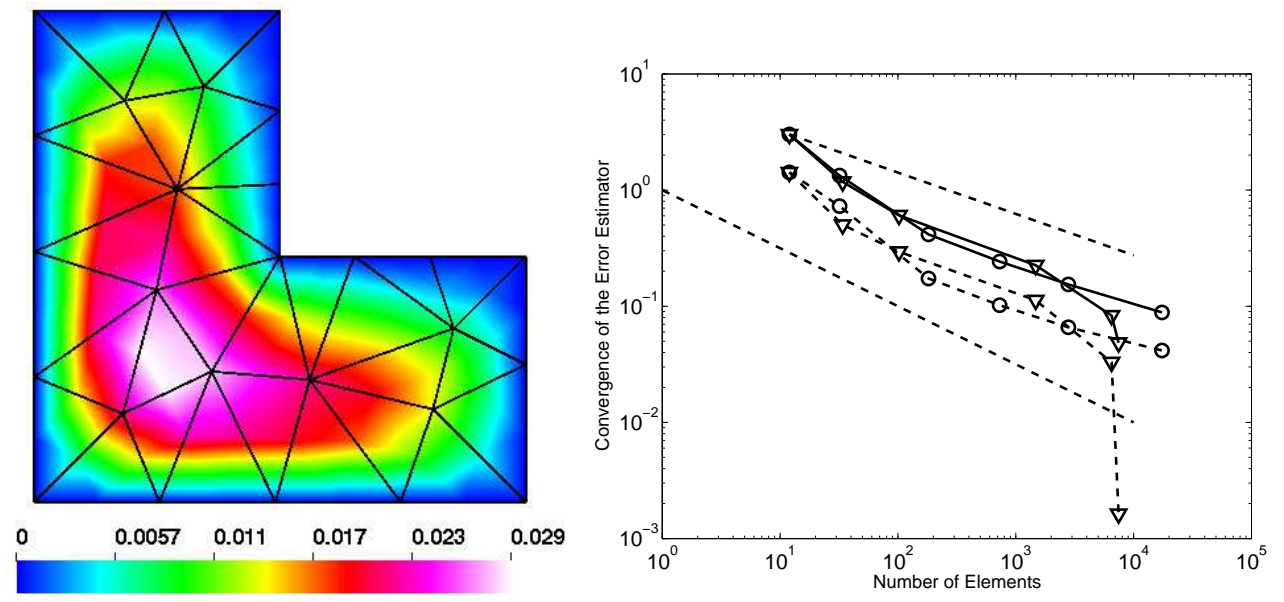

Figure 10: Clamped L-corner: Deflection distribution for the first mesh; Convergence of the global estimator (solid lines) and the maximum local estimator (dashed lines); Circles for the uniform refinements, triangles for the adaptive refinements.
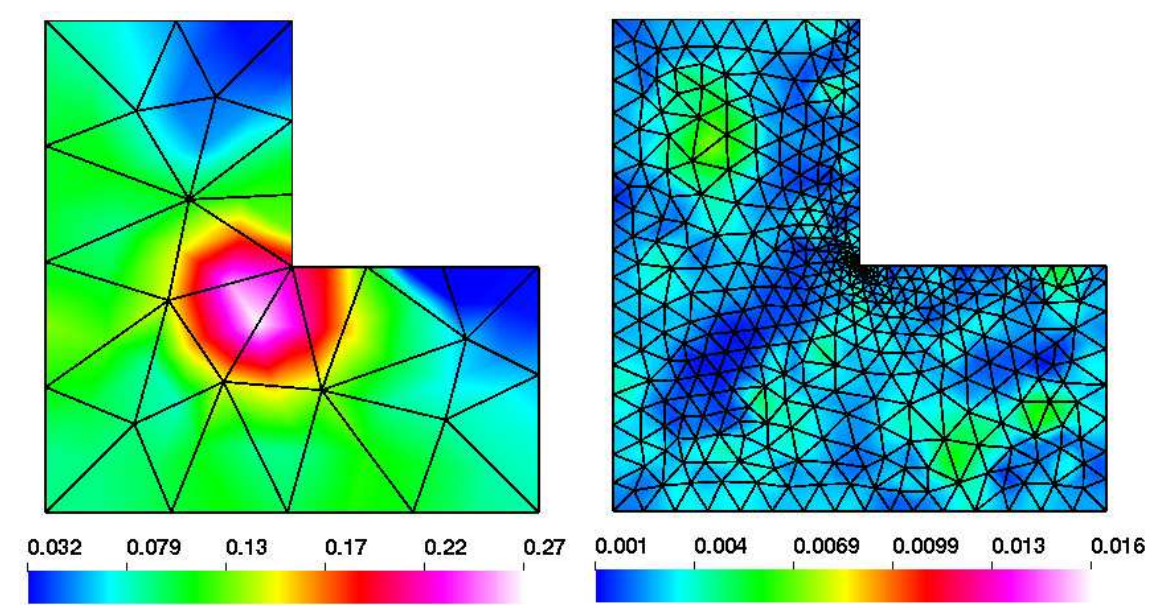

Figure 11: Clamped L-corner: Distribution of the error estimator for two different refinement steps. 

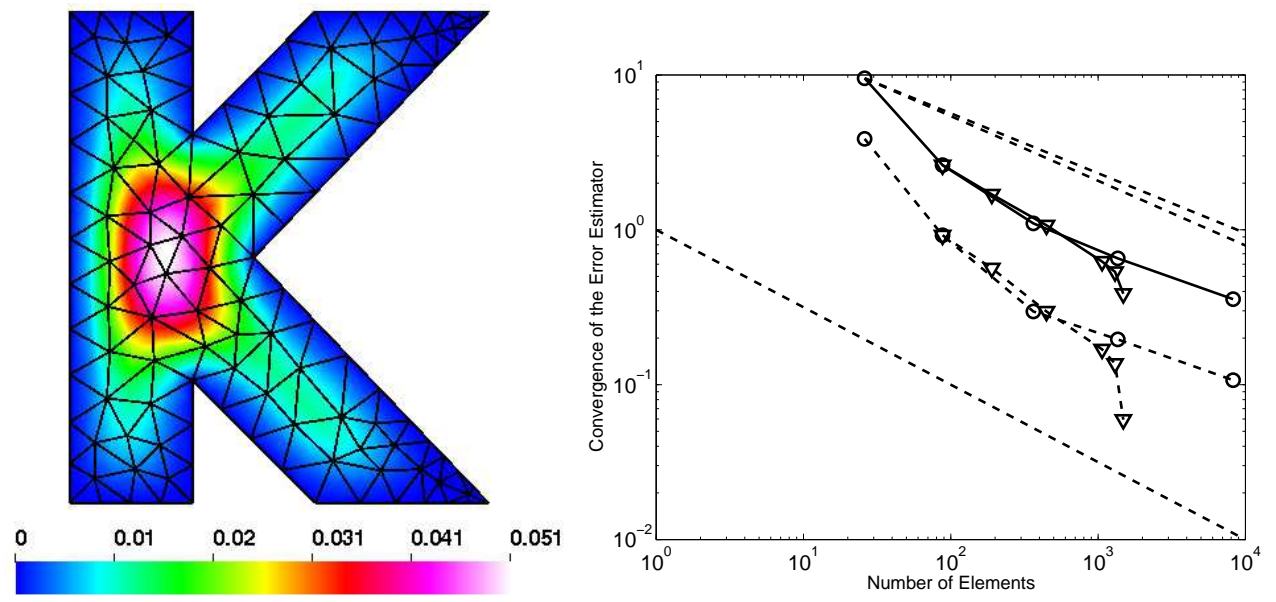

Figure 12: Clamped K-domain: Deflection distribution for the first mesh; Convergence of the global estimator (solid lines) and the maximum local estimator (dashed lines); Circles for the uniform refinements, triangles for the adaptive refinements.
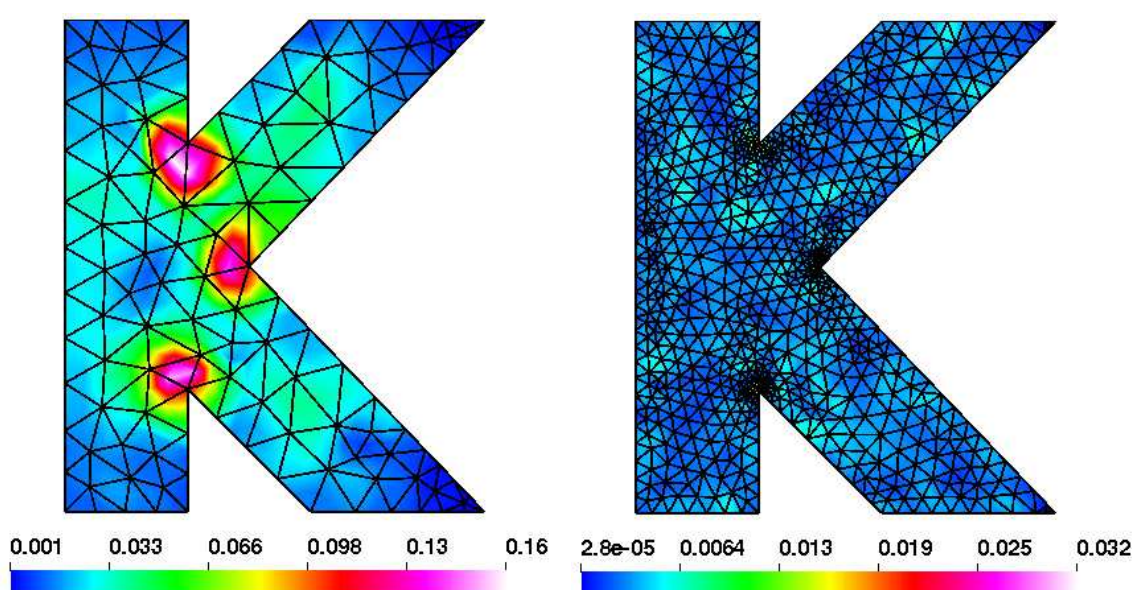

Figure 13: Clamped K-domain: Distribution of the error estimator for two different refinement steps. 

(continued from the back cover)

A516 Sergey Repin, Rolf Stenberg

Two-sided a posteriori estimates for the generalized stokes problem

December 2006

A515 Sergey Korotov

Global a posteriori error estimates for convection-reaction-diffusion problems

December 2006

A514 Yulia Mishura, Esko Valkeila

An extension of the L'evy characterization to fractional Brownian motion

December 2006

A513 Wolfgang Desch, Stig-Olof Londen

On a Stochastic Parabolic Integral Equation

October 2006

A512 Joachim Schöberl, Rolf Stenberg

Multigrid methods for a stabilized Reissner-Mindlin plate formulation

October 2006

A511 Carlo Lovadina, Mikko Lyly, Rolf Stenberg

A posteriori estimates for the Stokes eigenvalue problem

February 2007

A510 Janos Karatson, Sergey Korotov

Discrete maximum principles for FEM solutions of some nonlinear elliptic inter-

face problems

December 2006

A509 Jukka Tuomela, Teijo Arponen, Villesamuli Normi

On the simulation of multibody systems with holonomic constraints

September 2006

A508 Teijo Arponen, Samuli Piipponen, Jukka Tuomela

Analysing singularities of a benchmark problem

September 2006 


\section{HELSINKI UNIVERSITY OF TECHNOLOGY INSTITUTE OF MATHEMATICS}

RESEARCH REPORTS

The list of reports is continued inside. Electronical versions of the reports are available at $h t t p: / / w w w . m a t h . h u t . f i / r e p o r t s /$.

A522 Antti Hannukainen, Sergey Korotov, Marcus Ruter A posteriori error estimates for some problems in linear elasticity March 2007

A521 Sergey Korotov, Ales Kropac, Michal Krizek

Strong regularity of a family of face-to-face partitions generated by the longestedge bisection algorithm

April 2007

A519 Teemu Lukkari

Elliptic equations with nonstandard growth involving measure data

February 2007

A518 Niko Marola

Regularity and convergence results in the calculus of variations on metric spaces February 2007

A517 Jan Brandts, Sergey Korotov, Michal Krizek Simplicial finite elements in higher dimensions

February 2007 